\title{
caractérisation des non-linéarités apparues lors des essais de vibrations forcées en centrifugeuse
}

\author{
characterization of non-linearities occurred \\ during forced vibration tests in centrifuge
}

\author{
B. BOURDIN \\ Principia ${ }^{*}$ \\ M.P. LUONG - C.N.R.S. \\ L.M.S., Ecole Polytechnique* * \\ P. MORLIER \\ Université de Bordeaux $\mathrm{I}^{* *}$
}

Rev. Franç. Géotech. n 47 , p.p. $39-59$ (avril 1989)

\section{Résumé}

Nous décrivons les résultats obtenus lors des essais de vibrations sur des maquettes de pieux dans la centrifugeuse du CESTA. Nous essayons tout d'abord d'identifier les non-linéarités par une analyse détaillée des courbes dans les plans de Bode, de Nyquist ou de Nichols...

Nous proposons des interprétations théoriques actuellement développées : théorie des noyaux de Wiener, interpolation par les polynômes de Tchebycheff dans le plan de phase et exploitation de la partie non-causale par Villedieu.

\section{Abstract}

We describe some results obtained during forced vibration tests on pile models installed in the CESTA centrifuge. We first try to identify the nature of nonlinearities by a detailed analysis of curves in Bode, Nyquist or Nichols diagram...

We propose some theoretical interpretations which are being developed: Wiener kernel theory, phase plane interpolation by means of Tchebycheff polynom, non-causal part exploitation by Villedieu.

\footnotetext{
- Sophia Antipolis, B.P. 22, 06561 Valbonne.

*. 91128 Palaiseau Cedex.

*. 351. cours de la Libération, 33405 Talence Cedex.
} 


\section{INTRODUCTION}

Nous exposons, à la lumière de travaux récents, des méthodes de détection de non-linéarités rencontrées lors d'essais de vibrations sur fondations profondes ; nous tentons de mettre en évidence l'influence des lois de comportement sur les courbes fréquentielles grâce à une analyse détaillée des fonctions mesurées de réponse en fréquence.

Ces essais ont été réalisés sur des maquettes de groupes de pieux ou de pieux partiellement ancrés soumises à centrifugation.

L'interprétation sur maquettes est une méthode physique permettant la simulation du comportement d'un sol ou d'un système sol-structure.

Du fait de la complexité des lois de comportement des sols, caractérisées par la non-linéarité, l'écrouissage, le fluage, il apparaît nécessaire de réaliser un modèle réduit d'un système sol-fondation en conservant le matériau et les contraintes, c'est-à-dire tel que : - les contraintes en des points homologues du modèle et de la structure soient égales ;

- les lois rhéologiques en ces points soient, en conséquence, sensiblement les mêmes.

Lorsque le poids propre intervient, plusieurs procédés permettent la simulation des forces de masse pour satisfaire les conditions de similitude [10], en particulier la centrifugation sera utilisée dans ce qui suit.

\section{MOYENS D'ESSAIS}

\subsection{Caractéristiques de la centrifugeuse LATECOERE}

La centrifugeuse LATECOERE 265 du CESTA a été conçue pour simuler le comportement de spécimens pouvant avoir de grandes dimensions, soumis d'une part à une accélération statique, et d'autre part, éventuellement, à des conditions d'environnement variées : excitation vibratoire, conditionnement de température ou de vide.

La centrifugeuse comporte un bras dissymétrique d'une masse de 40 tonnes, équipé à 8,40 mètres de l'axe de quatre points d'ancrage permettant la fixation entre autres, d'une nacelle pivotante. Le bras repose sur un ensemble moyeu-support liés par un réducteur à la motorisation, qui est assurée par quatre moteurs à courant continu de $257 \mathrm{~kW}$ chacun.

Elle permet d'emporter des masses importantes en bout de bras : 2 tonnes à $100 \mathrm{~g}$ ou 4,6 tonnes à 43,5 $\mathrm{g}$ (g étant la gravité terrestre $\simeq 10 \mathrm{~m} / \mathrm{s}^{2}$ ).

Par ailleurs, les liaisons de mesure avec le modèle sont assurées par 92 cábles bifilaires blindés et 6 câbles coaxiaux, reliés avec le local de commande par des contacts tournants, à très faibles niveaux de bruit.

\subsection{Baie de pilotage}

La console de pilotage numérique 5427 A de Hewlett-Packard est un système spécialisé de pilotage de vibrations, en association avec un excitateur électrodynamique. Le mode de pilotage sinusoïdal du $5427 \mathrm{~A}$ permet de disposer d'un système numérique qui génère des ondes sinusoïdales, dont la fréquence balaie une plage allant de 0,1 à $5000 \mathrm{~Hz}$, grâce à un convertisseur numérique-analogique.

\subsection{Amplificateurs de puissance}

Les amplificateurs de puissance PRODERA A-438 peuvent délivrer un courant parfaitement imposé en amplitude et en phase. Cette caractéristique permet, dans lanalyse harmonique, d'exciter la structure par des forces dont l'amplitude et la phase sont indépendantes du mouvement; les caractéristiques sont :

- Gamme de la tension d'entrée : 0 - $5 \mathrm{~V}$ crête

- Courant maximum de sortie : 70 A crête

- Bande passante $\quad: 0-7000 \mathrm{~Hz}$

- Rapport signal-bruit

$6.10^{-4}$ pour le courant de sortie maximum

La stabilité de gain en fonction de la tension secteur est inférieure à $1.10^{-3}$ pour une variation de plus ou moins $10 \%$ de la tension du secteur.

\subsection{Excitateur électrodynamique}

L'excitateur utilisé est un excitateur PRODERA du type EX.420/D. Le principe de fonctionnement est le suivant :

- les courants d'excitation délivrés par les amplificateurs de puissance alimentent une bobine mobile se déplaçant, selon la loi de Laplace, au sein d'un champ magnétique généré par des bobines de champ. Afin d'assurer une excellente tenue de l'équipage mobile, face à la force centrifuge perpendiculaire à la direction d'excitation, la suspension de l'équipage mobile a été modifiée par l'adjonction de douilles à billes. Les caractéristiques techniques sont :

- Module maximum de force : $1000 \mathrm{~N}$

- Déplacement maximum

$: \pm 15 \mathrm{~mm}$

- Masse

$: 155 \mathrm{~kg}$

- Dimensions

: hauteur : $470 \mathrm{~mm}$ : diamètre : $360 \mathrm{~mm}$

Le refroidissement s'effectue par de l'air comprimé ; l'excitateur délivre sa force de $1000 \mathrm{~N}$ jusqu'à 400 $\mathrm{Hz}$.

Les figures 1 et 2 montrent le schéma d'un montage expérimental. 


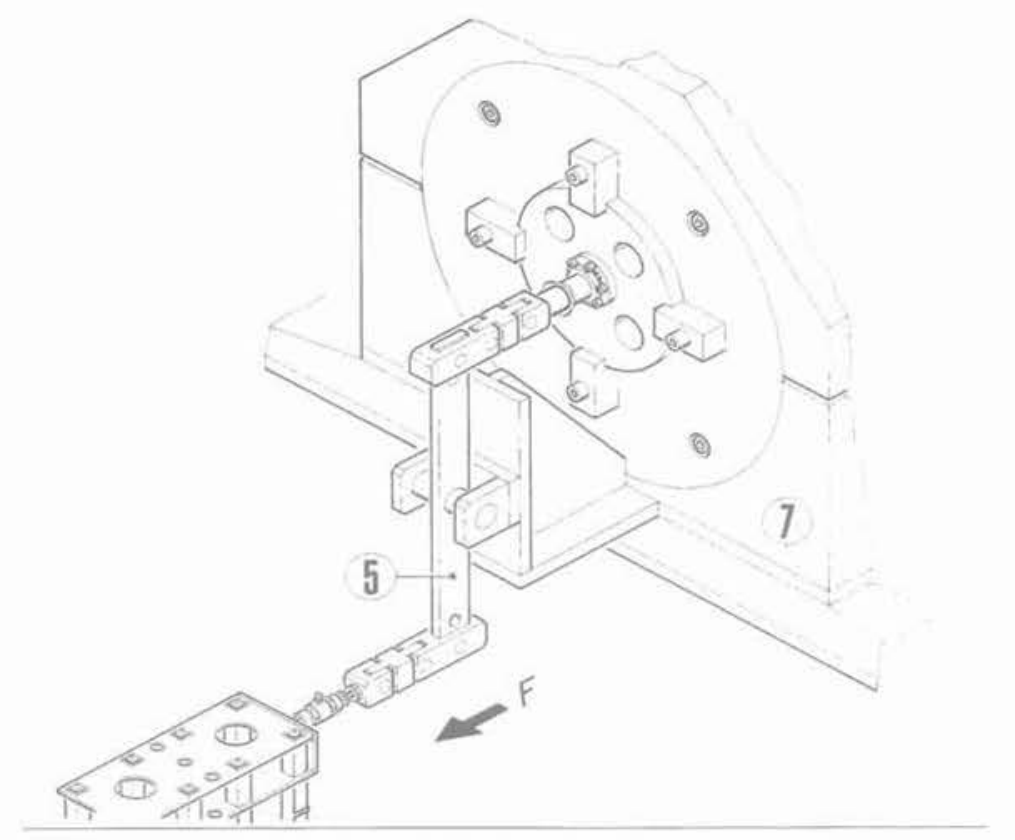

Fig. 1. - Détail du système d'excitation.

Fig. 1. - Detailed view of the cradle.

\section{MECANIQUE DES SOLS}

\section{SOLLIGITATIONS DYNAMIQUES}

EN GENTRIFUGEUSE

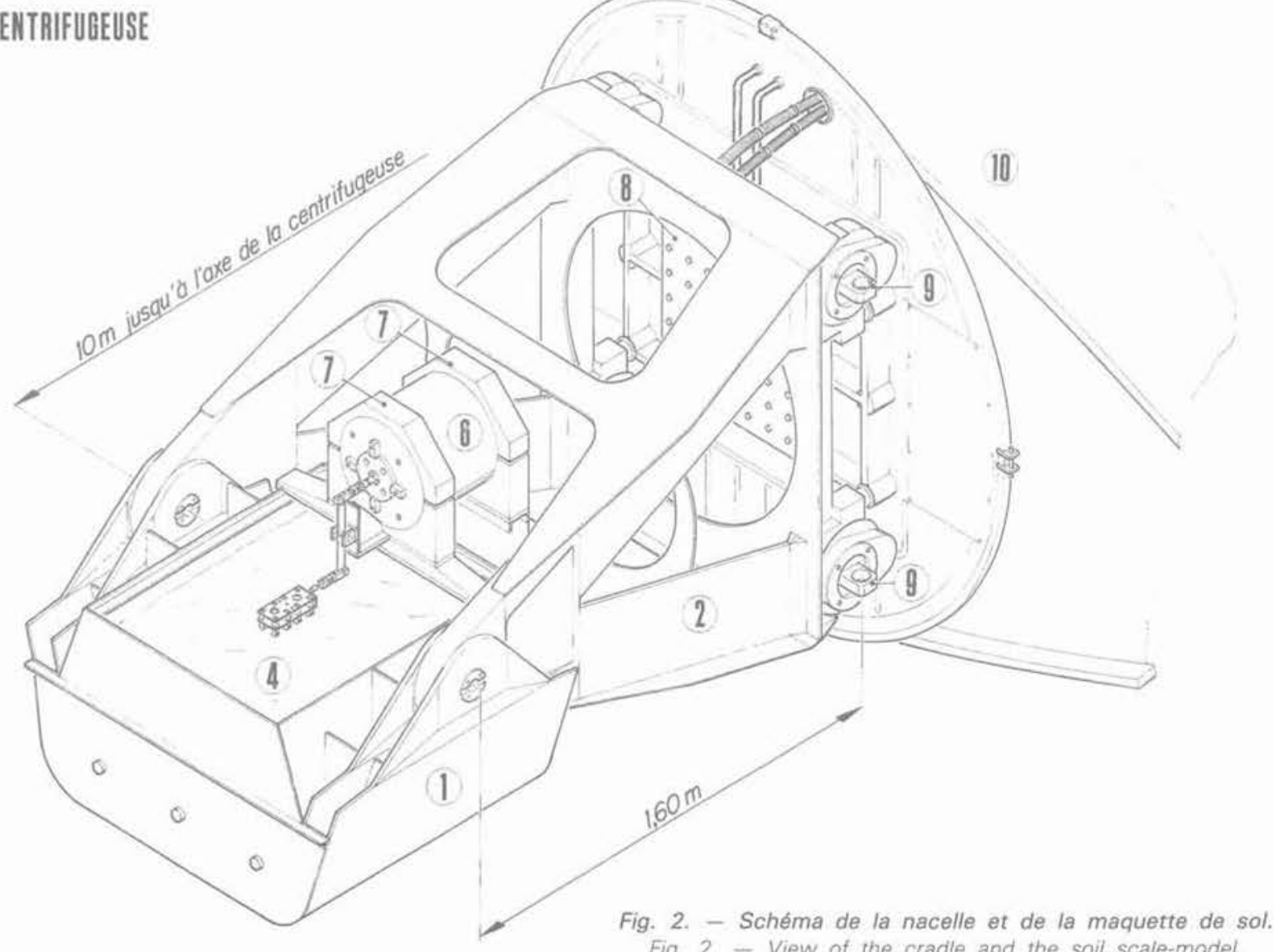

Fig. 2. - View of the cradile and the soil scale-model.
Fige 


\section{2. ÉTUDE EXPÉRIMENTALE}

\subsection{Conditions de sol en centrifugation}

Sable sec:

Une moyenne de six pesées a permis d'estimer la masse de sable embarqué à $337 \mathrm{~kg}$. La masse volumique déterminée est de l'ordre de $1400 \mathrm{~kg} / \mathrm{m}^{3}$; l'indice des vides $e_{0}$ est de l'ordre de 0,93. Des mesures du déplacement vertical en cours de centrifugation ont montré que le tassement était faible ; il est de l'ordre de $2 \mathrm{~mm}$ pour une épaisseur de sable de $38 \mathrm{~cm}$; en conséquence, l'indice des vides ne varie pratiquement pas.

Sable saturé :

Pour saturer le sable, nous avons introduit un tuyau au fond de la cellule, par lequel s'écoulait de l'eau à un très faible débit pendant 24 heures. La masse de sable saturé embarqué est de l'ordre de $472 \mathrm{~kg}$; la masse volumique estimée est alors de $1900 \mathrm{~kg} / \mathrm{m}^{3}$.

\subsection{Spécimens testés}

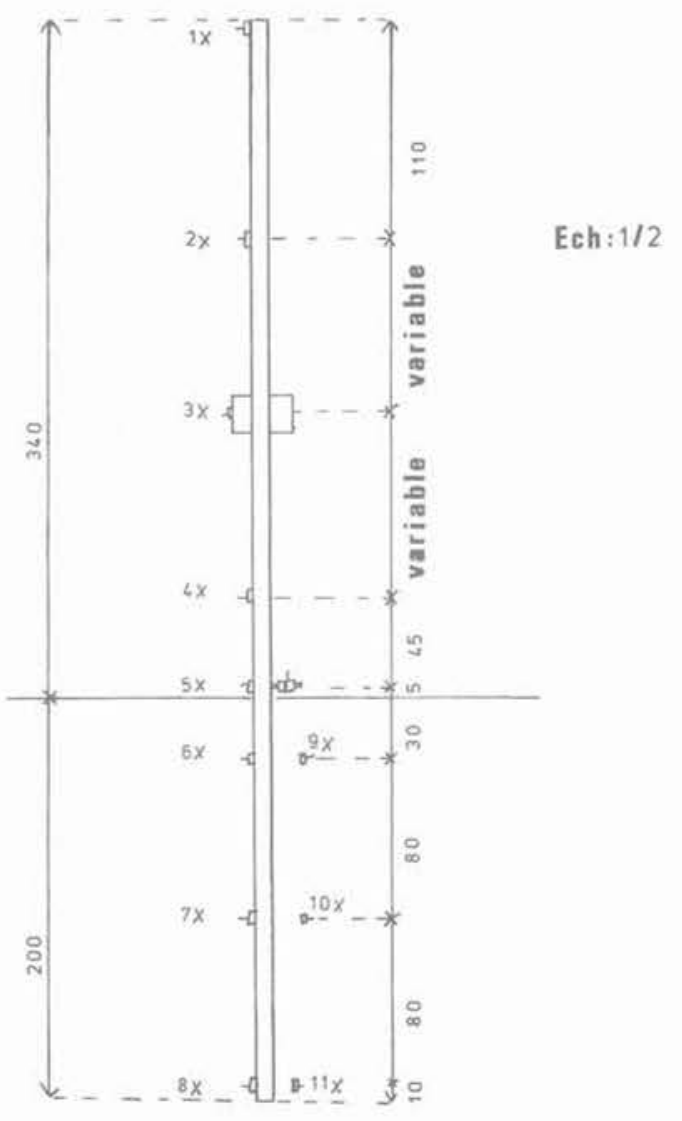

Fig. 3. - Spécimen en aluminium.

Fig. 3. - Aluminium testing specimen.
Le premier spécimen testé est un pieu en aluminium, partiellement ancré dans la maquette de sol (fig, 3).

Une masselotte en aluminium, de forme cylindrique, peut coulisser le long de la tige en aluminium de section circulaire. La hauteur de cette petite masse, que l'on peut régler à volonté, permet de faire varier les premiers modes de flexion du pieu.

L'instrumentation comporte cing accéléromètres sur la partie aérienne, trois dans la partie enterrée et trois autres placés à $15 \mathrm{~mm}$ de laxe du pieu en aluminium.

\subsection{Acquisition des signaux analogiques}

Tous les signaux ont été enregistrés sur bande magnétique au moyen d'un enregistreur Schlumberger 1600 , en modulation de fréquence. Ces enregistreurs utilisent un moteur central à circuit imprimé de faible inertie, sur laxe duquel est monté le cabestan. On supprime ainsi les courroies, cause de pleurage et de glissement. L'emploi de couloirs à dépression, placés entre les bobines et les circuits magnétiques, permet un défilement plus rigoureux devant les têtes. Le principe de l'enregistrement en modulation de fréquence est de transformer par un montage électronique une variation de tension en une variation de fréquence autour d'une fréquence centrale. L'emploi de ce procédé permet d'atteindre un taux de modulation de $40 \%$ avec une bonne linéarité.

Les caractéristiques de l'enregistrement sont :

- Largeur de bande : $25,4 \mathrm{~mm}$

- Vitesse de défilement de la bande : $19 \mathrm{~cm} / \mathrm{s}$

- Bande passante : $0 \mathrm{~Hz}-2500 \mathrm{~Hz}$

- Rapport signal/bruit $\quad: 46 \mathrm{~dB}$

Au début des essais, afin d'obtenir un codage correct en vue de la numérisation ultérieure des signaux. nous avons procédé à l'enregistrement d'une tension de calibration sur chaque piste de l'enregistreur à partir d'un signal de référence, identique pour les 14 voies d'enregistrement.

\subsection{Numérisation et traitement}

Tous les signaux sont relus à laide du même enregistreur et envoyés à un multiplexeur, dont chaque voie est munie d'un échantillonneur-bloqueur et d'un filtre anti-repliement.

Ces filtres sont constitués, pour chaque voie, de deux filtres passe-bas de Tchebycheff d'ordre 6 , placés en cascade. Leur atténuation est de $96 \mathrm{~dB} /$ octave audelà de la fréquence de coupure. La fréquence d'antirepliement est programmable par lutilisateur. La fonction temporelle filtrée subit un échantillonnage: la mémoire d'entrée permet le stockage de 1024 points temporels ; la fréquence d'échantillonnage était fixée à $1280 \mathrm{~Hz}$.

Par application de la transformée de Fourier discrète, les fonctions de réponse en fréquence calculées sont stockées sur 512 points ; la résolution de ces fonctions est de $1,25 \mathrm{~Hz}$. 
Le calcul de la transformée de Fourier discrète est fonction de la forme de la fenêtre d'observation. Pour atténuer la distorsion des spectres obtenus par application de la T.F.D., nous devons choisir judicieusement les fenêtres limitant la durée du signal; il faut que leur spectre possède des lobes latéraux aussi peu amples et aussi étalés que possible. La fenêtre rectangulaire est trop abrupte. Nous avons choisi la fenêtre de Hanning, définie dans le domaine temporel par :

$\Psi(t)=0,5\left(1+\cos 2 \pi \frac{t}{\theta}\right) 0 \leq t \leq \theta(2.1)$

où $\theta$ désigne la durée de la fenêtre temporelle.

La fonction de réponse peut être calculée directement en effectuant le rapport des transformées de Fourier des signaux d'accélération et de force; en fait, la méthode d'estimation utilisée effectue des opérations sur les spectres de puissance des signaux.

$G(\omega)=\frac{G_{y x}(\omega)}{G_{x x}(\omega)}$

$\mathrm{G}_{\mathrm{yx}}(\omega)$ représente les interspectres de puissance entre les signaux d'excitation et de réponse ; c'est la transformée de Fourier du produit de corrélation de $\mathrm{x}(\mathrm{t})$ avec $y(t)$.

$\mathrm{G}_{\mathrm{xx}}(\omega)$ représente l'autospectre de puissance du signal d'excitation ; c'est la transformée de Fourier du produit d'autocorrélation de $\mathrm{x}(\mathrm{t})$. Cette méthode d'estimation permet le calcul de la cohérence :

$\gamma_{y x}^{2}(\omega)=\frac{\left[G_{y x}(\omega)\right]^{2}}{G_{x x}(\omega) G_{y y}(\omega)}$

Si $\gamma^{2}=1$, il y a une causalité parfaite de la réponse par rapport à l'excitation et une absence totale de bruit.

Si $\gamma^{2}<1$, la réponse est en partie due au bruit environnant : c'est un critère de non-corrélation entre les signaux.

\subsection{Identification modale}

Les caractéristiques dynamiques d'une structure restent souvent une notion abstraite, liée au résultat d'un calcul par une méthode d'approximation variationnelle. Pour le spécialiste du calcul des structures, un mode est souvent assimilé aux notions de valeurs propres et de vecteurs propres d'un système matriciel, Dans notre analyse, un mode est considéré comme un pôle commun à toutes les inertances associées à notre système sol-pieu.

L'identification modale est l'ensemble des techniques permettant la détermination des paramètres modaux d'une structure : fréquences propres, amortissements modaux et déformées modales.

Dans le cas d'une excitation en un seul point, la fonction de réponse en fréquence mesurée est considérée comme la somme de fractions rationnelles dont chacune exprime la réponse d'un mode propre:

$$
\begin{aligned}
& G(\omega)=-\frac{1}{\mu \omega^{2}}+\sum_{r=1}^{n} \\
& \frac{A^{r}}{\omega_{T}^{2}-\omega^{2}+2 j \omega \omega_{r} \xi_{T}}+C
\end{aligned}
$$

$\mathrm{n}$ : Nombre de modes dont la pulsation se trouve dans la bande de fréquence étudiée,

$\mathrm{C}$ : Influence des modes d'ordre supérieur,

$\mu$ : Influence des modes d'ordre inférieur et des mouvements de corps rigide,

$\mathrm{A}^{r}$ : Amplitude du mode $r$ pour un point d'excitation et le point de mesure fixés,

$\xi_{r}$ : Amortissement visqueux équivalent.

A partir des fonctions de réponse en fréquence mesurées, nous utilisons l'algorithme MDOF ( Multi Degree of Freedom ») pour extraire les paramètres modaux: fréquences, amortissements, amplitude et déphasage : le principe de cette technique repose, dans le domaine temporel, sur l'analyse de la transformée inverse de Fourier de la fonction de réponse en fréquence $G(\omega)$ : la "réponse impulsionnelle " est ainsi décomposée en une série d'exponentielles complexes.

A l'aide de cette technique d'identification, nous avons analysé les courbes fréquentielles issues de tous les capteurs.

\subsection{Représentation d'une fonction à valeurs complexes}

Les rapports accélération sur force, ou inertance, mesurés sont, dans le domaine des fréquences, des fonctions à valeurs complexes de la forme :

$G(\omega)=\operatorname{Re}(\omega)+j \operatorname{Im}(\omega)$

où $\operatorname{Re}(\omega)$ désigne la partie réelle de $G(\omega)$, et $\operatorname{Im}(\omega)$ est la partie imaginaire de $\mathrm{G}(\omega)$.

- Représentation de Bode :

$G(\omega)$ peut être exprimée par :

$\mathrm{G}(\omega)=|\mathrm{G}(\omega)| e^{\jmath \phi}$

On exprime alors le module

$|G(\omega)|=\sqrt{\operatorname{Re}^{2}(\omega)+\operatorname{Im}^{2}(\omega)}$ et la phase

$\Phi=\tan ^{-1} \frac{\operatorname{lm}(\omega)}{\operatorname{Re}(\omega)}$ en fonction de la pulsation $\omega$.

- Représentation de Nyquist:

La partie imaginaire $\operatorname{Im}(\omega)$ est exprimée en fonction de la partie réelle.

- Représentation de Nichols :

Le module $[G(\omega) \mid$ est exprimé en fonction de la phase. 


\section{3. ÉTUDE DES NON-LINÉARITÉS}

La présence de caractéristiques non-linéaires dans le système sol-fondation provoque sur les courbes fréquentielles mesurées des distorsions qui rendent hasardeuse la pratique de lanalyse modale sur un tel système. Basée sur l'algèbre linéaire, l'analyse modale ne convient pas à la description des systèmes nonlinéaires; la validité de l'identification modale peut être gravement compromise.

L'étude expérimentale d'un système non-linéaire, dont le modèle mathématique n'est pas connu a priori, peut s'effectuer en trois phases:

- la détection;

- la caractérisation :

- la quantification.

Nous présentons plusieurs méthodes de détection qui sont du ressort de l'analyse des signatures:

- distorsion des diagrammes de Nyquist ;

- transformée de Hilbert.

L'étude des harmoniques n'est pas évoquée.

La caractérisation est effectuée par comparaison des courbes expérimentales et des courbes issues de simulations de non-linéarités. Des méthodes sont proposées en vue d'une estimation quantitative.

\subsection{Comparaison entre la fonction de réponse en fréquence mesurée et la fonction générée à partir des paramètres modaux}

\subsubsection{Plan de Bode}

La superposition, dans le plan de Bode, de la courbe fréquentielle mesurée et de la courbe reconstituée à partir des paramètres modaux extraits par MDOF laisse à penser que les valeurs des amplitudes $\mathrm{A}^{t}$ des inertances sont correctement évaluées. Ceci n'est vrai que dans le domaine des faibles valeurs de sollicitations effectuées sur nos essais (fig. 4 et 5 ).

Des essais de vibrations sur pieux, réalisés par M.P. LUONG à un fort niveau de sollicitation, montrent que la superposition des courbes n'est plus possible (fig. 6).

La courbe de principe 7 laisse supposer l'existence d'une gamme de force au-delà de laquelle l'influence du comportement non-linéaire est très importante.

\subsubsection{Plan de Nyquist}

Dans le plan de Nyquist, les écarts dans le cas de faibles sollicitations se manifestent; la courbe mesurée a une forme aplatie et protubérante laissant croire à la présence de plusieurs modes; la courbe calculée à partir des paramètres modaux est une ellipse, ce qui traduit un amortissement visqueux équivalent important, de l'ordre de $3 \%$. Rappelons que dans le cas d'un amortissement hystérétique, nous obtenons théoriquement des cercles modaux parfaits, que la fonction mesurée soit une réceptance, une mobilité ou
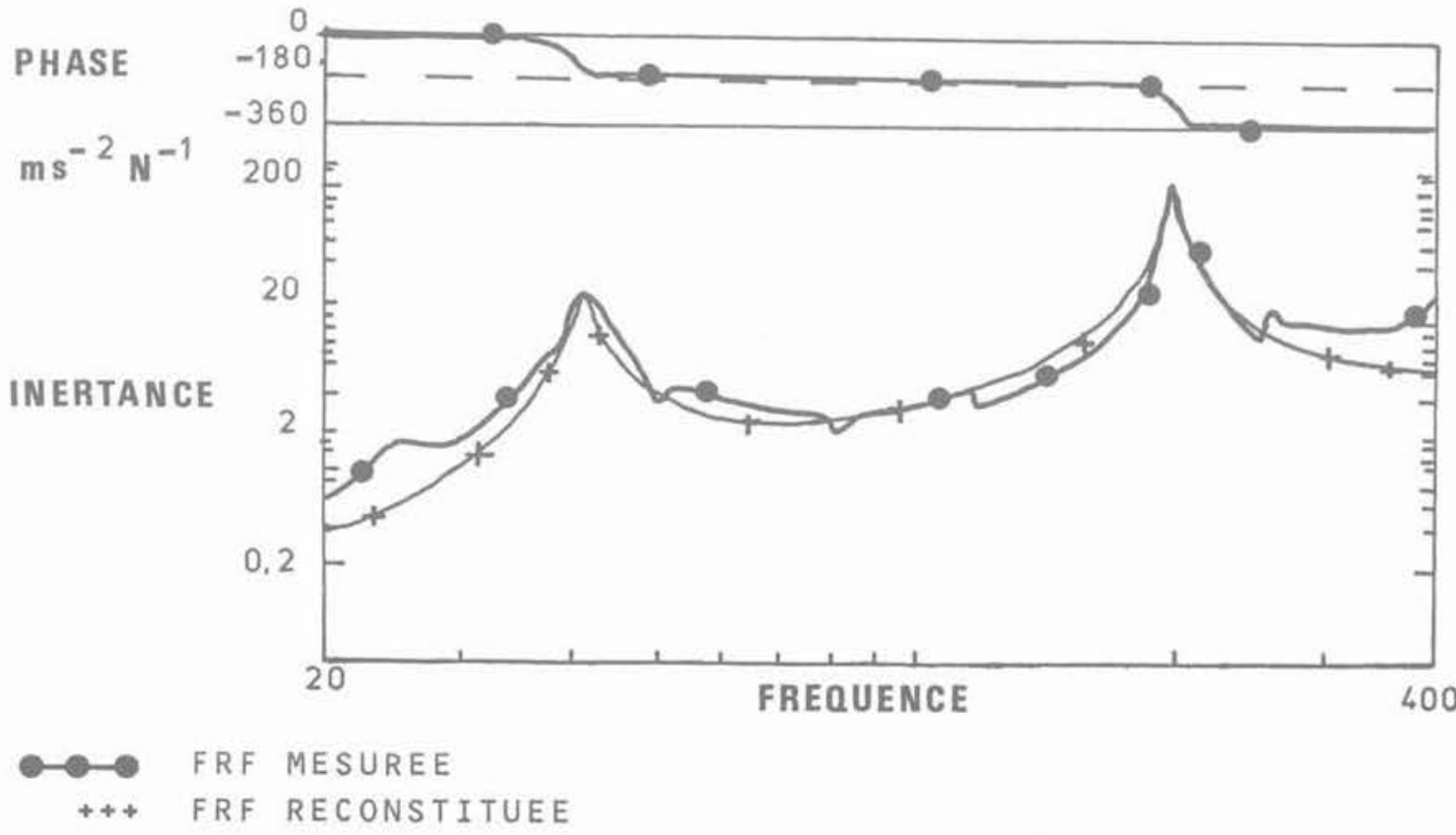

Fig. 4. - Superposition des courbes mesurées et reconstituées pieu-aluminium $H=14$ sable sec. Fig. 4. Measured and reconstructed curves superimposition aluminium-pile $H=14$ dry sand. 


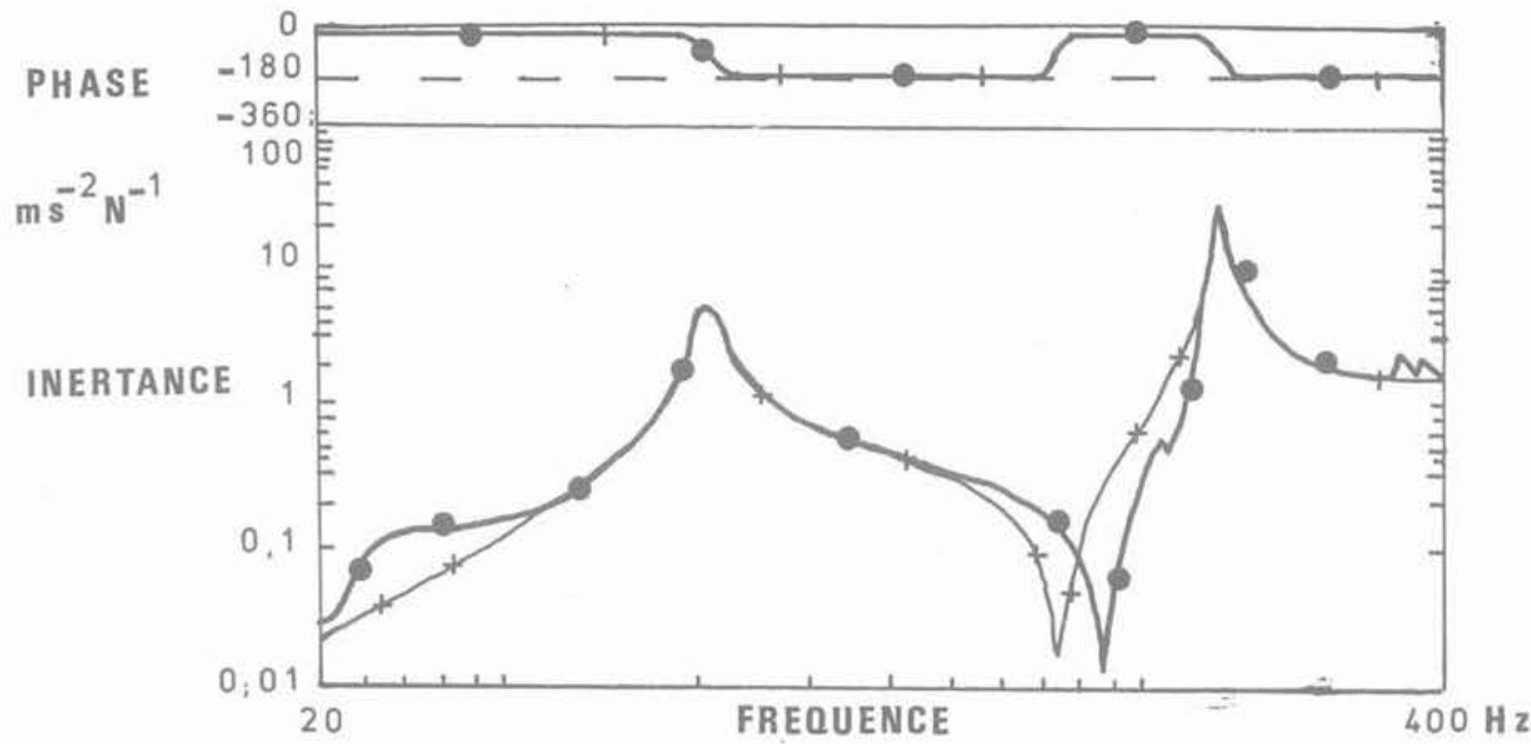

$\begin{array}{ll}- & \text { FRF MESUREE } \\ +++ & \text { FRF RECONSTITUEE }\end{array}$

Fig. 5. - Superposition des courbes mesurées et reconstituées pieu acier-sable saturé Fig. 5. - Measured and reconstructed curves superimposition steel pile-saturated sand.

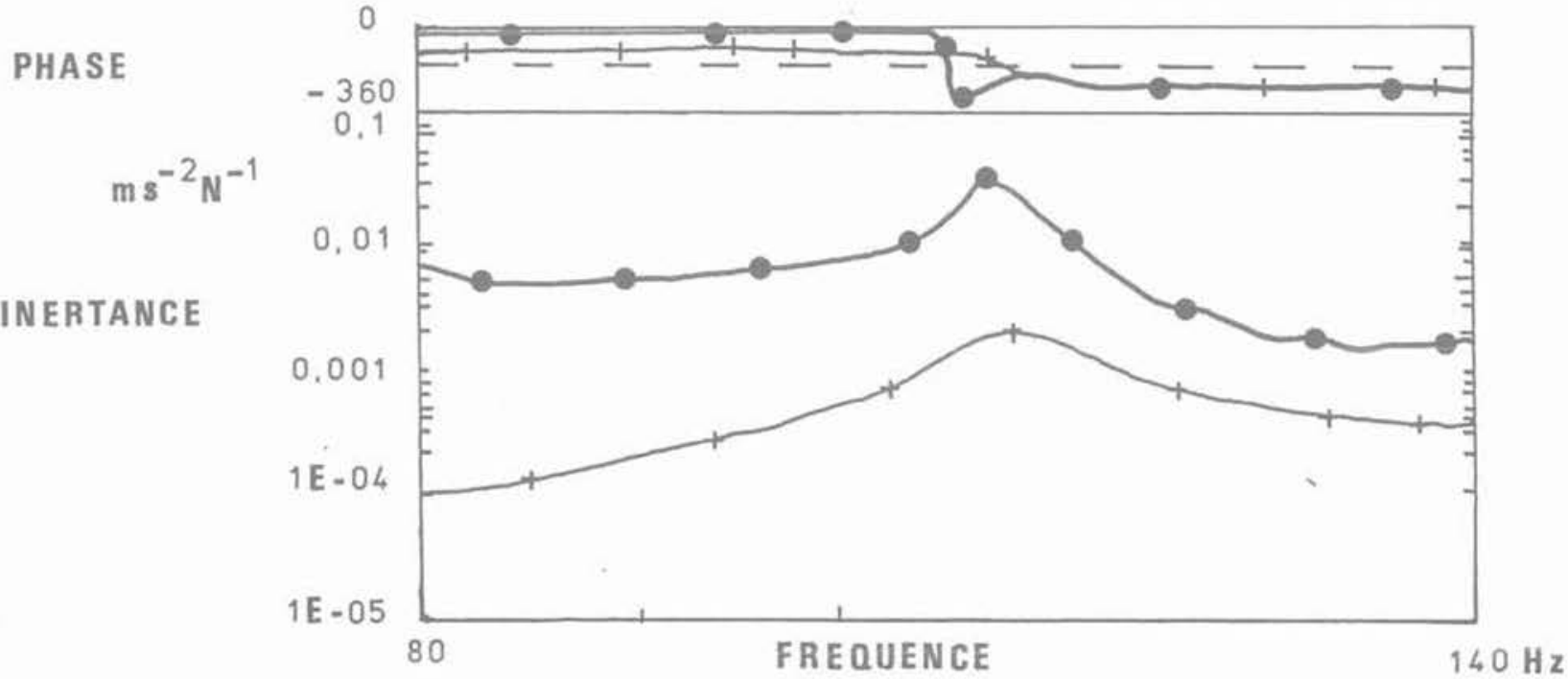

\footnotetext{
- FRF MESUREE

+++ FRF RECONSTITUEE
}

Fig. 6. - Superposition de la FRF mesurée et de la FRF reconstituée (essai Luong $F=300 \mathrm{~N}$ capteur $3 X$ ). Fig. 6. - Measured and reconstructed FRF superimposition (Luong test $F=300 \mathrm{~N}-3 \times$ transductor). 


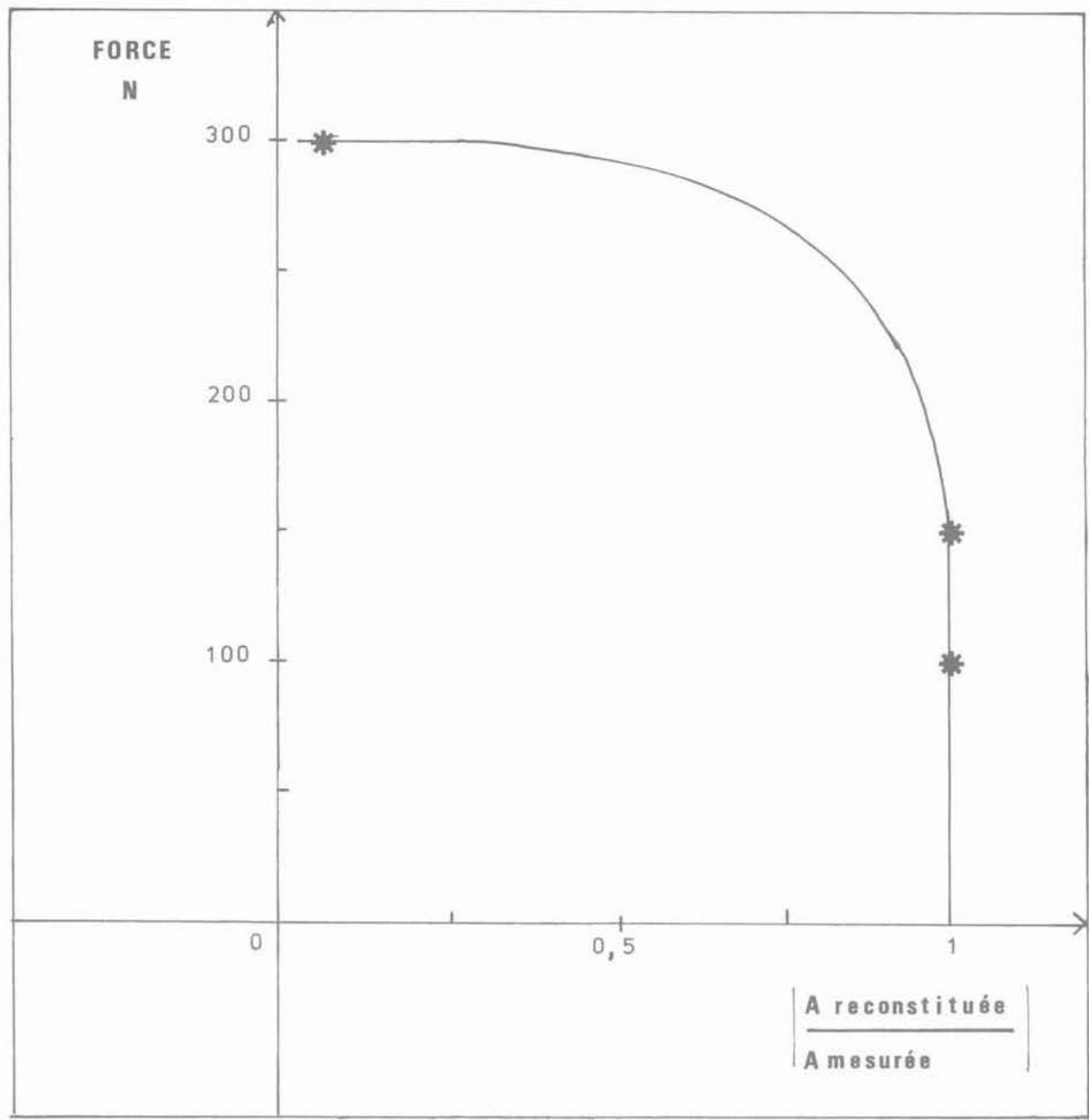

Fig. 7. - Courbe de principe montrant que pour une gamme de force, le comportement non-linéaire est important (essai Luong, 1 pieu).

Fig. 7. - Curve which shows that for a force range, the non-linear behaviour is important (Luong test, single pile).

une inertance ; par contre, ceci n'est valable pour un amortissement visqueux que dans le cas d'une mobilité.

Ainsi, la méthode d'identification modale, selon un modèle linéaire, n'est pas réalisable à partir des courbes fréquentielles obtenues par une excitation sinusoï- dale sur les modèles de fondation que nous avons étudiés.

Rappelons que la réceptance est le rapport déplacement sur force, la mobilité désigne le rapport vitesse sur force et l'inertance est le rapport accélération sur force. 


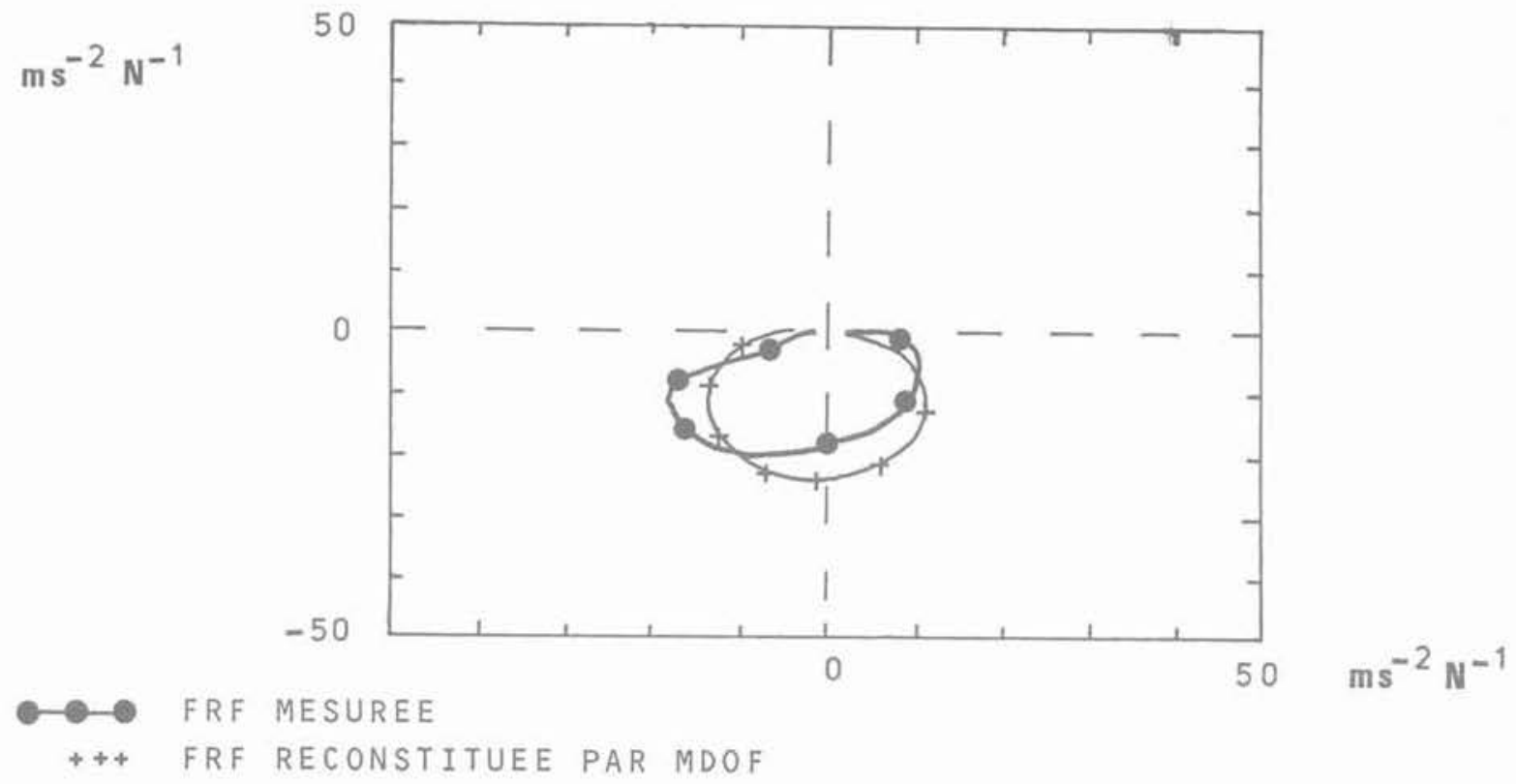

$$
\begin{aligned}
& \text { Fig. 8. - Lieu de Nyquist: } \\
& \text { FRF mesurée- } \\
& \text { FRF reconstituée par MDOF. } \\
& \text { Fig. } 8 \text { - Nyquist plot: } \\
& \text { Measured FRF. } \\
& \text { MDOF reconstructed FRF. }
\end{aligned}
$$

\subsection{Observations et interprétation} sur la transformée inverse de Fourier; application de la transformée de Hilbert

Lorsque l'on calcule la transformée inverse de Fourier d'une fonction de réponse en fréquence, on s'attend à obtenir une réponse temporelle entièrement située dans l'intervalle de temps positif. En pratique, l'application de la transformée inverse de Fourier discrète sur la partie mesurée de la fonction de réponse en fréquence revient à remplacer les modes à haute fréquence par des modes qui ont les mêmes caracté. ristiques d'amplitude et d'amortissement que les modes à basse fréquence. Par conséquent, la réponse temporelle ainsi calculée peut différer sensiblement de la réponse impulsionnelle d'un système même parfaitement linéaire. D'autre part, l'utilisation de la transformée de Fourier discrète fait translater les éventuelles parties non-causales qui devraient figurer dans les temps négatifs, dans la deuxième moitié de l'intervalle de temps considéré.

La figure 9 montre la transformée inverse de Fourier d'une fonction de réponse issue de nos expériences en sable saturé. Elle présente des pseudo-sinusoïdes avec des enveloppes exponentielles décroissantes pour la partie causale et des pseudo-sinusoïdes avec une enveloppe exponentielle croissante pour la partie non-causale.

\subsubsection{Relations entre non-causalité et non-linéarité}

La méthode des éléments finis en mécanique des milieux continus repose sur le calcul variationnel utilisant des fonctionnelles.
Une fonctionnelle est donnée par la relation :

$J(y)=\int_{x_{1}}^{x_{2}} F(x, y, \dot{y}, \ddot{y}) d x$

Entre deux points $\left(x_{1}, y_{1}\right),\left(x_{2}, y_{2}\right), J(y)$ dépend du chemin que prend $y(x)$ entre les points $x_{1}$ et $x_{2}$.

La fonctionnelle décrit tout phénomène physique dépendant de l'histoire du chargement $\mathrm{x}(\mathrm{t})$, de la vitesse $\dot{x}(t)$ et de son accélération $\dot{x}(t)$. Pour les systèmes mécaniques non-linéaires, dont la réponse dépend non seulement de l'histoire du chargement mais aussi du niveau de l'excitation, on conçoit que les fonctionnelles constituent un cadre mathématique approprié pour l'étude de non-linéarités.

Les travaux développés dans la littérature par FRECHET [6], HAOUI [7] et SCHETZEN [14] sont développés.

- Développement en série de Volterra de la réponse d'un système physique:

VOLTERRA, en 1880, généralisa les séries de Taylor s'appliquant aux fonctions continues, aux fonctionnelles continues. En 1910, FRECHET [6] démontra que toute fonctionnelle continue peut être représentée par une série de fonctionnelles d'ordres entiers, série dont la convergence est uniforme dans tout ensemble compact de fonctions continues. 


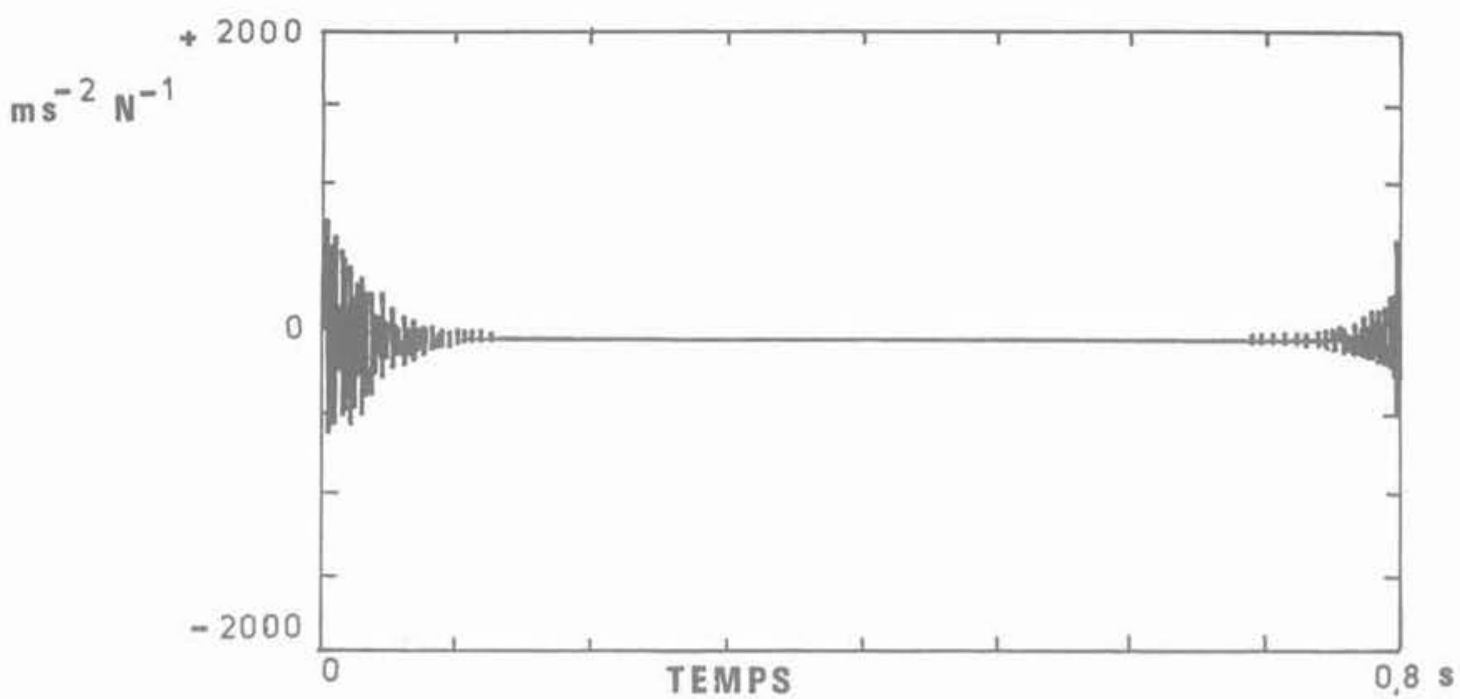

Fig. 9. - Transformée inverse de Fourier $g(t)$ de la fonction $g(\omega)$ mesurée.

Fig. 9. $-g(t)$ Fourier Inverse Transform of $G(w)$ measured function.

La réponse $y(t)$ est traduite par une convolution linéaire entre l'entrée $\mathrm{x}(\mathrm{t})$ et la réponse dite impulsionnelle du système $h_{1}(t)$ :

$y(t)=\int_{-\infty}^{+\infty} h_{1}(\tau) \times(t-\tau) d \tau$

On étend la convolution aux systèmes non-linéaires en faisant appel à une série dont le premier terme est égal à l'expression (3.2) et dont les termes suivants font appel aux convolutions multiples :

$$
\begin{aligned}
& y(t)=\int_{-\infty}^{+\infty} h_{1}(\tau) x\left(t-\tau_{1}\right) d \tau_{1} \\
& +\int_{-\infty}^{+\infty} \int_{-\infty}^{+\infty} h_{2}\left(\tau_{1}, \tau_{2}\right) \times\left(t-\tau_{1}\right) \times\left(t-\tau_{2}\right) d \tau_{1} d \tau_{2} \\
& +\ldots+\int_{-\infty}^{+\infty} \ldots \\
& \int_{-\infty}^{+\infty} h_{p}\left(\tau_{1}, \tau_{2}, \ldots, \tau_{p}\right) \times\left(t-\tau_{1}\right) \\
& x\left(t-\tau_{2}\right) \ldots x\left(t-\tau_{p}\right) d \tau_{1} d \tau_{2} \ldots d \tau_{p}
\end{aligned}
$$

Cette représentation a été utilisée récemment à l'ordre deux en hydrodynamique pour l'étude des efforts du second ordre sur une structure soumise à une houle aléatoire [5].

\section{- Conséquence de cette hypothèse} sur la transformée inverse de Fourier, HAOUI [7] :

Dans la relation $(3.3), h_{p}\left(\tau_{1}, \tau_{2}, \ldots, \tau_{p}\right)$ est le $p^{\text {tème }}$ noyau de Volterra; il est interprété comme une réponse impulsionnelle à $\mathrm{p}$ dimensions.
La causalité du système non linéaire est assurée si et seulement si :

$h_{p}\left(\tau_{1}, \tau_{2}, \ldots, \tau_{p}\right)=0 \tau_{j}<0 j=1,2, \ldots, p$

Posons :

$y(t)=\sum_{p=1}^{n} y_{p}(t)$

où :

$$
\begin{aligned}
& y_{p}(t)=\int_{-\infty}^{+\infty} \ldots \int_{-\infty}^{+\infty} \\
& h_{p}\left(\tau_{1}, \ldots, \tau_{p}\right) \times\left(t-\tau_{1}\right) \ldots x\left(t-\tau_{p}\right) d \tau_{1} \ldots d \tau_{p}
\end{aligned}
$$

SCHETZEN [14] a montré, en utilisant la transformée de Fourier multidimensionnelle, que la représentation spectrale du p ième opérateur de Volterra peut s'écrire sous la forme :

$$
\begin{aligned}
& Y_{p}(f)=\int_{-\infty}^{+\infty} \ldots \int_{-\infty}^{+\infty} \\
& \tilde{h}_{p}\left(f-f_{1}, f_{1}-f_{2}, \ldots, f_{p-1} d f_{1} \ldots d f_{p-1}\right.
\end{aligned}
$$

$h_{p}$ représente la transformée de Fourier à $p$ dimensions du $\mathrm{p}^{\text {ième }}$ noyau de Volterra.

$\bar{h}_{p}\left(f_{1} \ldots, f_{p}\right)=$

$\int_{-\infty}^{+\infty} \ldots \int_{-\infty}^{+\infty} h_{p}\left(\tau_{1}, \ldots, \tau_{p}\right) e^{-12 \pi\left(f_{1} \tau_{1}+\ldots+f_{p} \tau_{p}\right)} \mathrm{d} \tau_{1} \ldots d \tau_{p}$ 
La fonction de réponse en fréquence est donnée par la relation :

$G(f)=\frac{\sum_{p=1}^{n} y_{p}(f)}{X(f)}$

$G(f)=\sum_{D=1}^{n} \frac{1}{X(f)}\left[\int_{-\infty}^{+\infty} \cdots \int_{-\infty}^{+\infty}\right.$

$\tilde{h}_{p}\left(f-f_{1}, f_{1}-f_{2}, \ldots . f_{p-1}\right)$

$\left.X\left(f-f_{1}\right) X\left(f_{1}-f_{2}\right) \ldots X\left(f_{p}-1\right) d f_{1} d f_{2} \ldots d f_{p-1}\right]$

La transformée inverse de Fourier unidirectionnelle s'écrit :

$g(t)=\sum_{p=1}^{n} \int_{-\infty}^{+\infty} \ldots \int_{-\infty}^{+\infty} \tilde{h}_{p}\left(f_{1}, f_{2}, \ldots, f_{p}\right) X\left(f_{2}\right) \ldots X\left(f_{p}\right)$

$\frac{e^{i 2 \pi\left(f_{1}+f_{2}+\ldots f_{p}\right) t}}{X\left(f_{1}+f_{2}+\ldots+f_{p}\right)} d f_{1} \ldots d f_{p}$

On remarque que cette fonction dépend de $\mathrm{X}(\mathrm{t})$ donc de la nature de l'excitation $x(t)$.

- Cas particulier idéal :

Prenons une distribution de Dirac comme signal d'excitation :

$$
\begin{aligned}
& x(t)=\delta\left(t-t_{0}\right) \\
& x(f)=e^{-i 2 \pi t_{0}}
\end{aligned}
$$

Portons la relation (3.13) dans (3.11):

$g(t)=\sum_{p=1}^{n} h_{p}(t, t, \ldots, t)$

$g(t)$ est causal d'après $(3,4)$.

- Cas général

$S \mathrm{x}(\mathrm{t})$ n'est pas une impulsion, la fonction $\mathrm{g}(\mathrm{t})$ est, en général, non-causale et n'a pas de sens physique d'après VINH [16].

\section{Exemple :}

$\mathrm{Si}$ :

$x(t)=\delta(t)+\delta\left(t-t_{0}\right)$

$X(f)=1+e^{-12 \pi f t o}$

$$
\begin{aligned}
& \mathrm{g}(\mathrm{t})=\sum_{p=1}^{n} \int_{-\infty}^{+\infty} \ldots \int_{-\infty}^{+\infty} \\
& \frac{\bar{h}\left(f_{1}, \ldots f_{p}\right)\left[1+e^{-2 i \pi f_{1} t_{0}}\right] e^{2 i \pi\left(f_{1}+\ldots+f_{p}\right)}}{1+e^{-2 i \pi\left(f_{1}+\ldots+f_{p}\right)} t_{0}} d f_{1} \ldots d f_{p}
\end{aligned}
$$

En s'arrêtant au deuxième ordre dans la série, le résultat pouvant être généralisé, nous avons :

$g(t)=h_{1}(t)+h_{2}(t, t)+$

$\sum_{m=0}^{\infty}\left(h_{2}\left(t+2 m t_{0}, t+(2 m+1) t_{0}\right)\right.$

$\left.-h_{2}\left(t+(2 m+1) t_{0}, t+2(m+1) t_{0}\right)\right\}$

Pour $\mathrm{t}<-\mathrm{p} \mathrm{t}_{0}$, nous avons :

$\left.g(t)=\sum_{m=n}^{\infty} h_{2}\left[t+2 m t_{0}, t+(2 m+1) t_{0}\right)\right]$

La fonction $\mathrm{g}(\mathrm{t})$, a priori, n'est pas causale.

Sous réserve d'admettre la représentativité du comportement des systèmes non-linéaires par les séries de Volterra, et de généraliser le résultat obtenu dans le cas de deux impulsions, nous pouvons considérer que la fonction définie comme la transformée d'un système non-linéaire est non-causale quand le signal d'excitation diffère d'une distribution de Dirac, d'après les travaux effectués par HAOUI [7].

\subsubsection{Application de la transformée de Hilbert}

La transformée de Hilbert est connue dans son principe par les spécialistes de l'automatique et de l'électricité ; les premières applications de cette transformée à la dynamique des structures ont été faites en France par VINH en 1984 [16].

La transformée de Hilbert est définie par l'intégrale de Cauchy:

$H[G(\omega)]=-\frac{1}{j \pi} V P \int_{-\infty}^{+\infty} \frac{G(\Omega)}{\Omega-\omega} d \Omega$

où VP désigne la valeur principale de Cauchy.

Dans le domaine temporel, on obtient, par application de la transformée inverse de Fourier:

$$
F^{-1}\{H[G(\omega)]\}=(2 U(t)-1) \cdot g(t)
$$

$\mathrm{U}(\mathrm{t})$ est la fonction échelon unitaire de Heaviside ; on ramène donc la transformée de Hilbert à un produit 


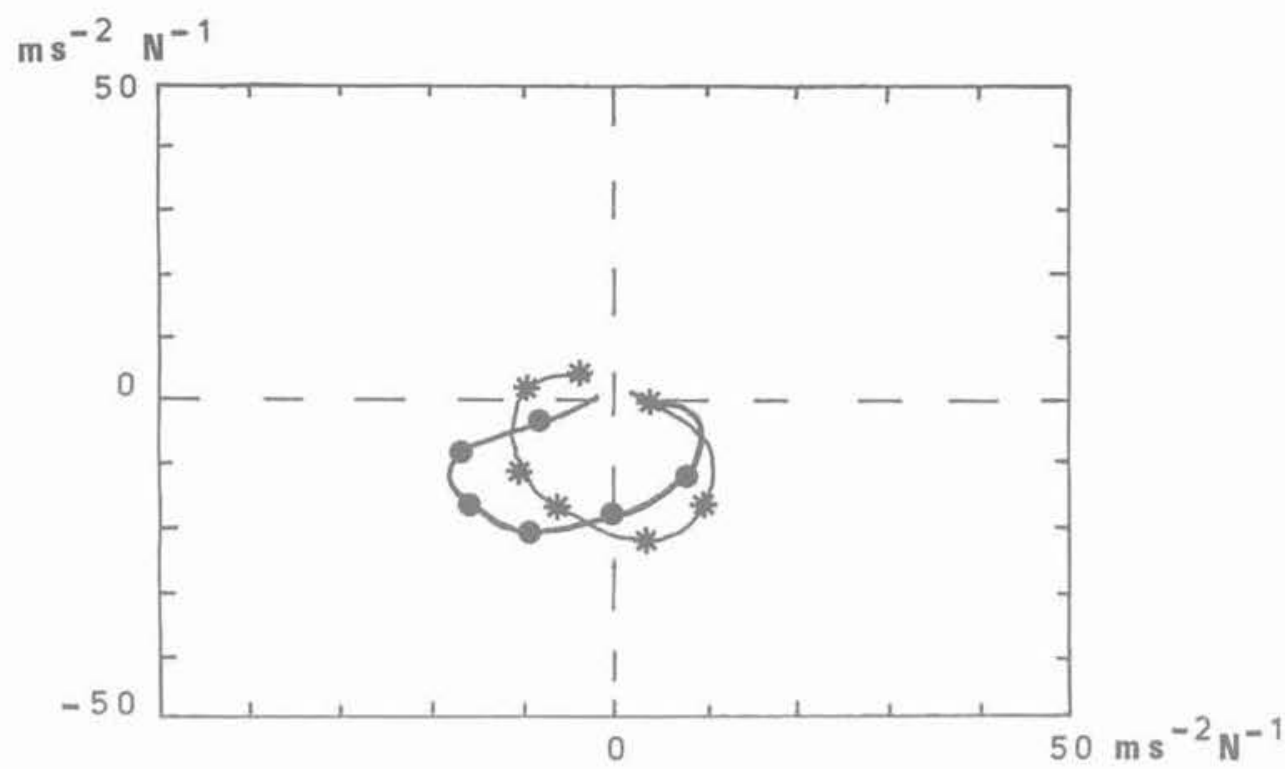

G $-(\omega)$ MESUREE

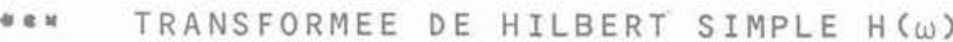

Fig. 10. - Lieu de Nyquist:

G(w) mesurée

Transformée de Hilbert simple $H(\omega)$.

Fig. 10. - Nyquist plot

Measured $G(\omega)$

Simple Hilbert transform $H(\omega)$.

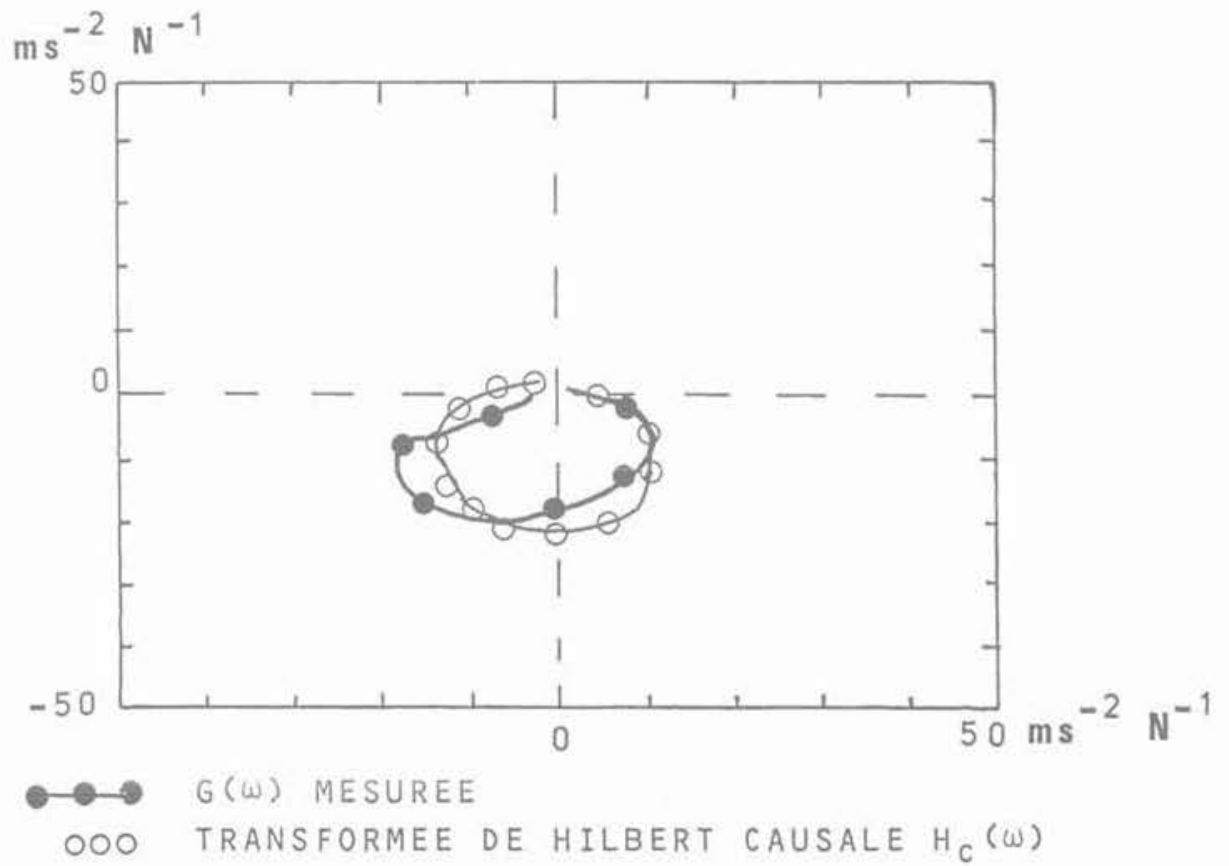

Fig. 11. - Lieu de Nyquist:

G(w) mesurée

Transformée de Hilbert causale $H_{c}(\omega)$.

Fig. 11. - Nyquist plot

Measured $G(\omega)$ -

Causal Hilbert Transform $\mathrm{H}_{C}(\omega)$. 


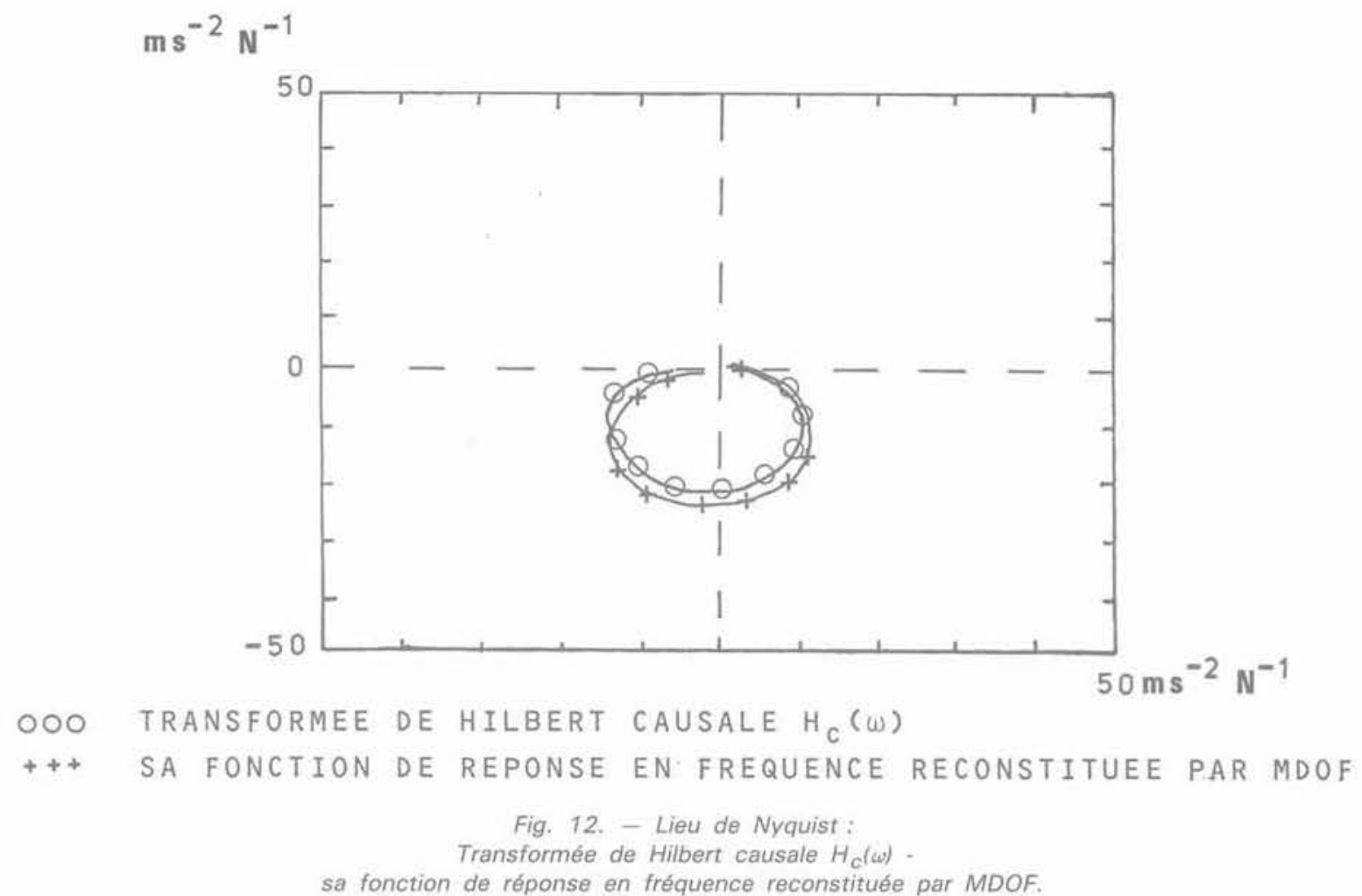

sa fonction de réponse en fréquence reconstituée par MDOF.

Fig. 12 - Nyquist plot:

Causal Hilbert transform $\mathrm{H}_{\mathrm{c}}(\omega)$.

its MDOF reconstructed frequency response function.

emporel simple, l'application de cette transformation changeant le signe de la partie non-causale.

HAOUI [7], en vertu de cette propriété, formule le critère de linéarité suivant :

- pour un système causal, si $G(\omega)$ désigne la fonction de réponse en fréquence pour une excitation différente d'une impulsion et si $H(\omega)$ est la transformée de Hilbert de $G(\omega)$, alors nous pouvons juger de la linéarité du système de la manière suivante :

si $H(\omega)=G(\omega)$, le système est linéaire ou à comportement linéaire pour l'excitation considérée ;

si $H(\omega) \neq G(\omega)$, le système est non-linéaire.

La figure 10 montre la superposition dans le plan de Nyquist de la fonction de réponse en fréquence mesurée avec sa transformée de Hilbert pour les mêmes données que la figure 8 . D'après le critère précédent, la structure est donc non-linéaire.

\subsubsection{Linéarisation par la transformée de Hilbert causale}

HAOUI [7] définit la transformée de Hilbert causale $\mathrm{H}_{\mathrm{c}}(\omega)$ :

$$
H_{c}(\omega)=\frac{1}{2}[G(\omega)+H(\omega)]
$$

La figure 11 montre la fonction $G(\omega)$ mesurée et sa transformée de Hilbert causale $\mathrm{H}_{c}(\omega)$. La transformée de Hilbert causale efface évidemment les distorsions et protubérances de la fonction mesurée. Comme la méthode MDOF, la transformée de Hilbert causale permet d'obtenir un système équivalent au système étudié ; elle s'applique à tout système mécanique pour lequel il n'est pas nécessaire de connaître, a priori, la nature des non-linéarités, même s'il est fortement non-linéaire.

L'extraction à l'aide de MDOF s'applique de façon satisfaisante à la transformée de Hilbert causale de nos résultats expérimentaux (fig. 12).

\subsection{Tentatives d'identification des non-linéarités}

Un certain nombre d'auteurs ont étudié l'influence des non-linéarités de comportement sur la réponse d'une structure approchée par un oscillateur à un degré de liberté :

$m \ddot{x}+C(\dot{x}) \dot{x}+k(x) x=f(t)$

Ce paragraphe va être consacré à l'analyse des signatures dans le domaine fréquentiel.

- Non-linéarité liée à la variation de la raideur

OKUBO [12] utilise une courbe "effort-déplacement " de la forme:

$k(x)=k_{0}\left(1+\beta x^{2}\right)$ 


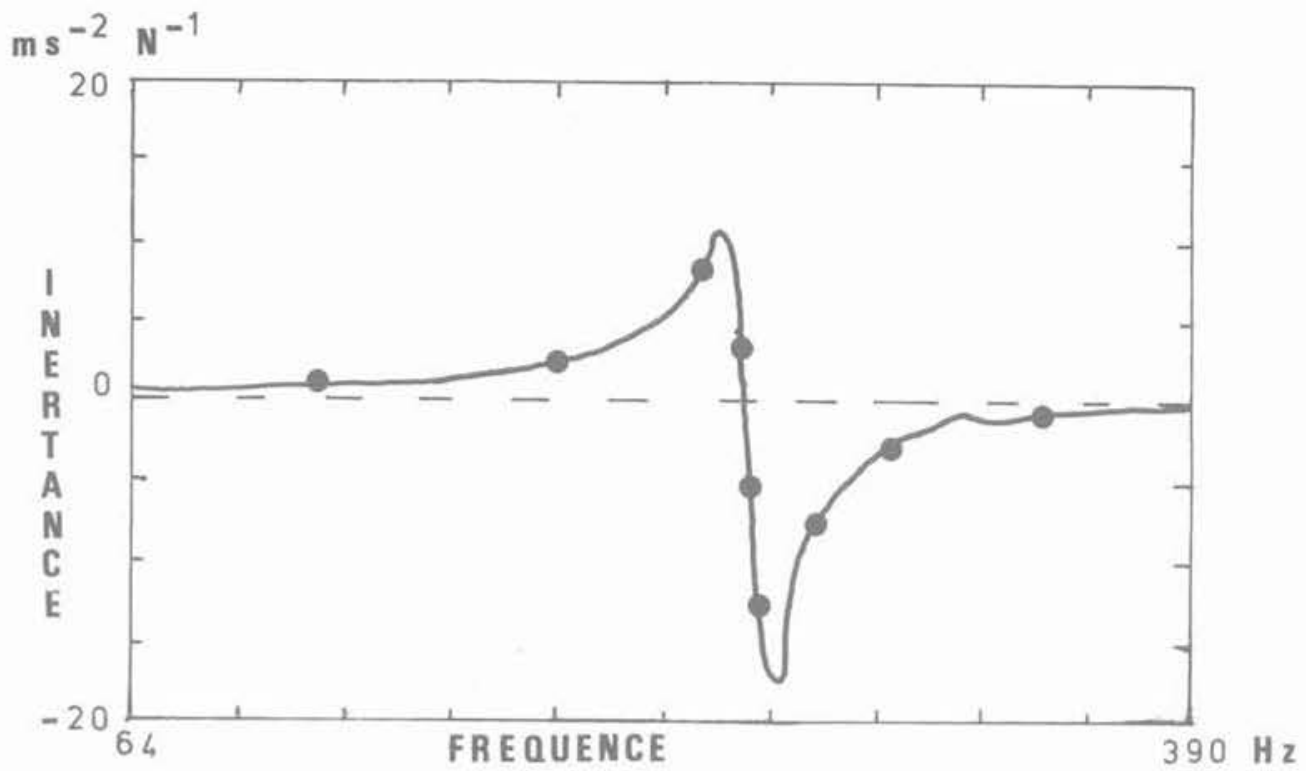

Fig. 13. - Partie réelle de $G(\omega)$ mesurée - Inertance.

Fig. 13. - Measured Gl $\omega$ real part - Inertance.

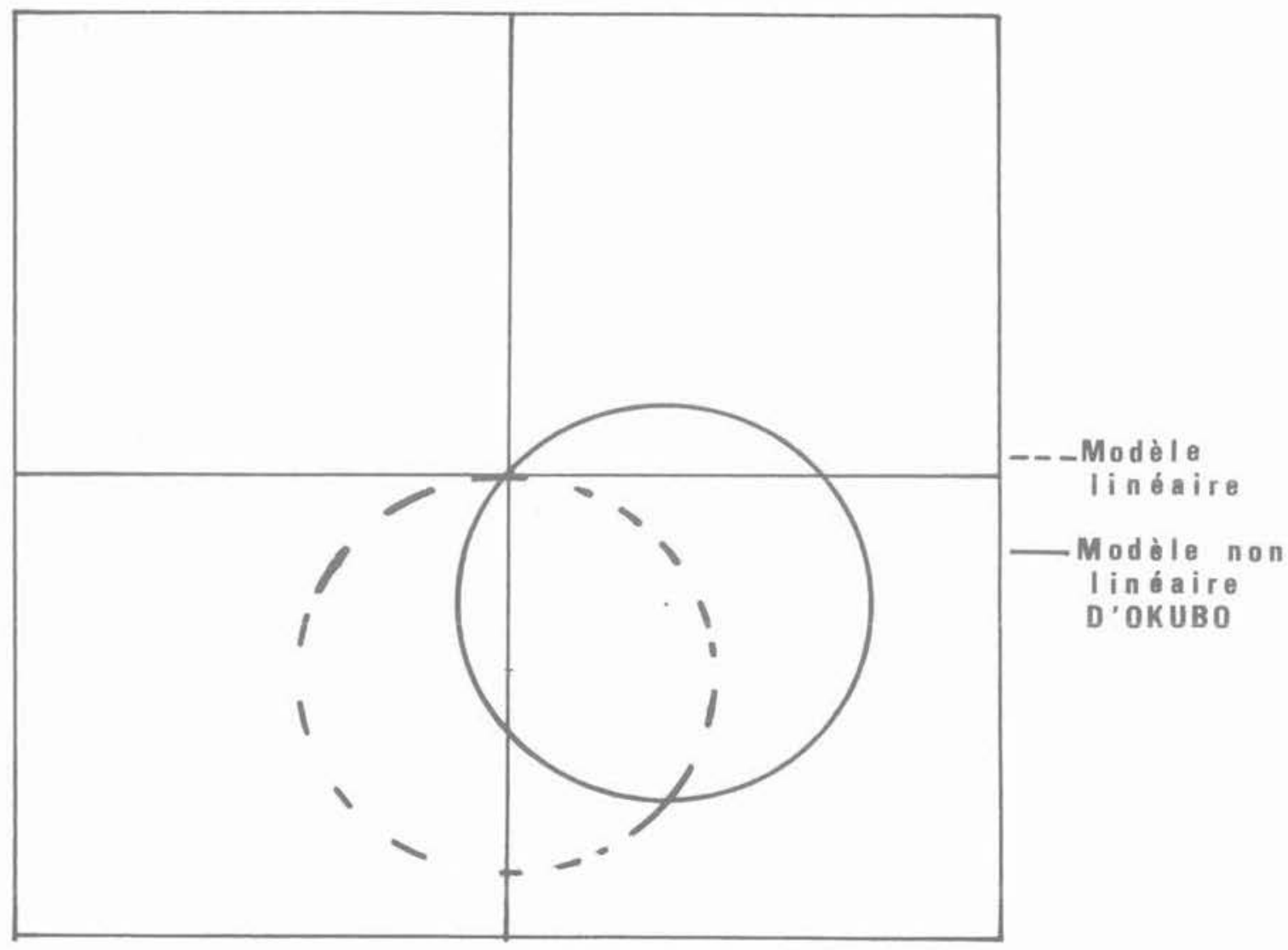

Fig. 14. - Rotation de la réponse $G(\omega)$ d'après OKUBO: Modèle linéaire

Modèle non-linéaire d'Okubo.

Fig. 14. - Response $G(\omega)$ rotation after OKUBO: Linear model

Okubo non-linear model. 


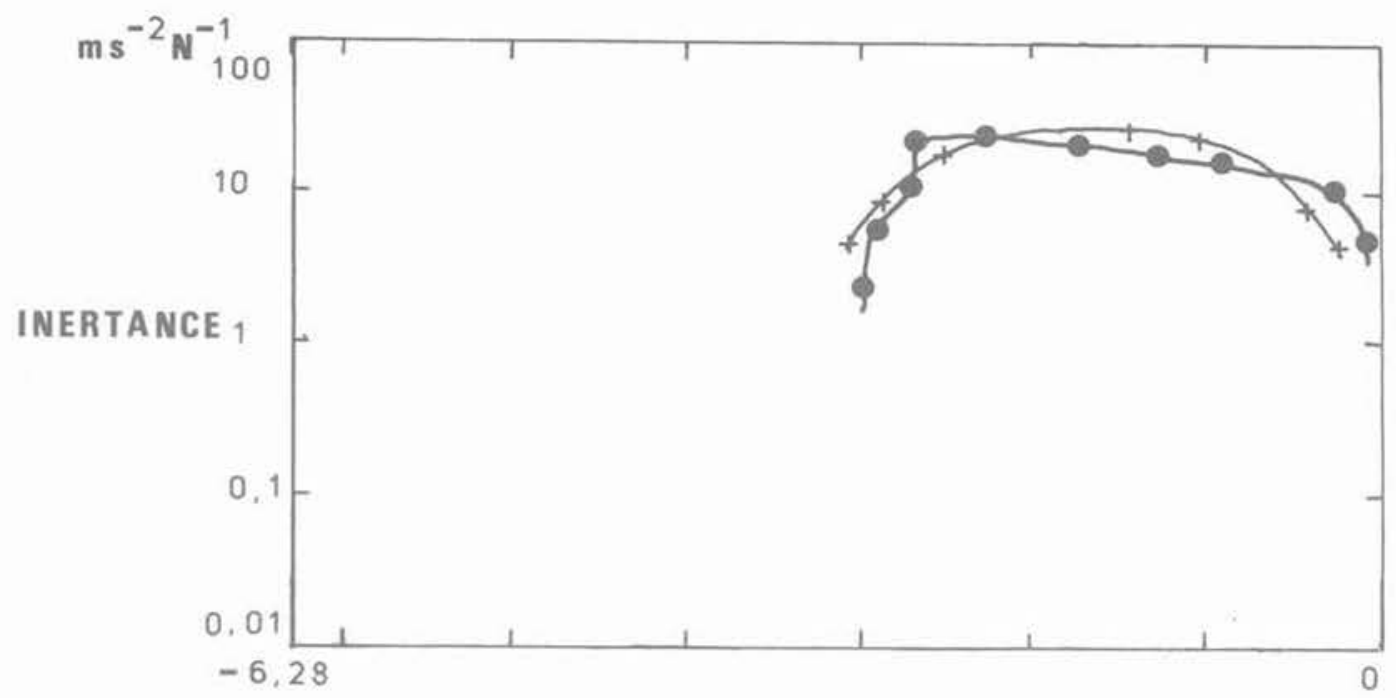

Phase radian

Fig. 15. - Représentation de Nichols.

Fig. 15. - Nichols plot.

Les conséquences de ce modèle de raideur sont les suivantes:

- une dissymétrie de la partie réelle de la réceptance autour de la fréquence de résonance. Par analogie, la figure 13 montre la dissymétrie obtenue dans les courbes « inertance-fréquence * issues de nos mesures (second mode de flexion du pieu);

- une rotation de la courbe de Nyquist qui croît avec la valeur du paramètre $\beta$ (fig. 14).

LALLEMENT [9] ajoute que les courbes de Nyquist restent des cercles dans ce cas, mais la phase ne tourne pas de façon symétrique de part et d'autre de la pulsation propre.

La présentation de Nichols, où l'on exprime le module en fonction de la phase, met en évidence cette rotation non-symétrique ; on remarque que la fonction reconstituée à partir des paramètres modaux conserve la symétrie (fig. 15).

NORMAN [12] a calculé la réponse d'un système régi par une loi de comportement élasto-plastique ; il constate alors la naissance de pseudo-modes qui apparaissent comme la sormme de modes très couplés.

\section{- Non-linéarité liée à la variation de l'amortissement}

LALLEMENT a étudié un amortissement $c(\dot{x})$ en $\dot{\mathrm{x}}^{3}$; il note que cette non-linéarité déforme les lieux de Nyquist qui, restant symétriques par rapport à l'axe des imaginaires, deviennent elliptiques.

OKUBO a supposé, dans un de ses modèles, que l'amortissement change de valeur lorsque la vitesse $\dot{x}$ dépasse un seuil : les lieux de Nyquist trouvent alors une forme ovoïde.

Les conclusions de ces auteurs sont issues d'expériences de simulation de non-linéarités soit par des méthodes analogiques, soit par des méthodes numériques.

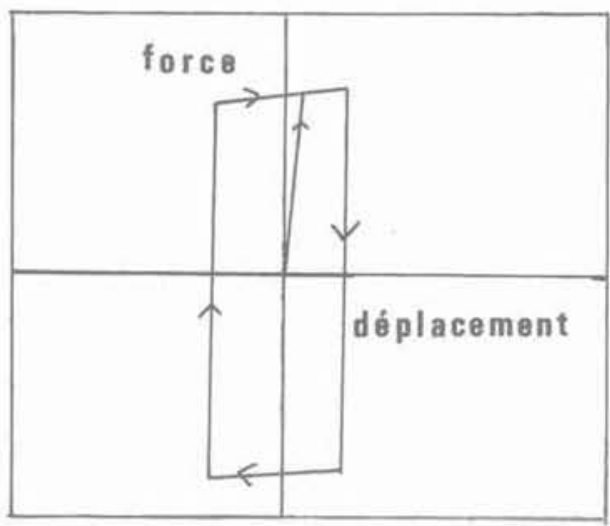

Fig. 16. - Plasticité - Loi étudiée par Norman en dynamique. Fig. 16. - Plasticity - Law which has been studied by Norman in dynamic problem.

\section{Application au système pieu-sol}

Pour les résultats obtenus sur la figure 13, la dissymétrie de la partie réelle observée sur nos fonctions et la rotation non symétrique de la phase autour de la résonance dans le plan de Nichols laissent supposer une non-linéarité de raideur; la forme elliptique de la figure 8 traduit une non-linéarité d'amortissement; la présence d'une protubérance dans cette même figure pourraît être la conséquence d'une loi de comportement plastique, comme celle adoptée par NORMAN. Les résultats s'appliquent indifféremment aux résultats de vibrations sur le pieu en sable saturé et en sable sec.

Ces conclusions sont purement qualitatives ; dans le but de mettre en œuvre des méthodes plus performantes d'identification des non-linéarités, nous proposons un ensemble de stratégies possibles en vue d'une modélisation plus rigoureuse du phénomène physique. 


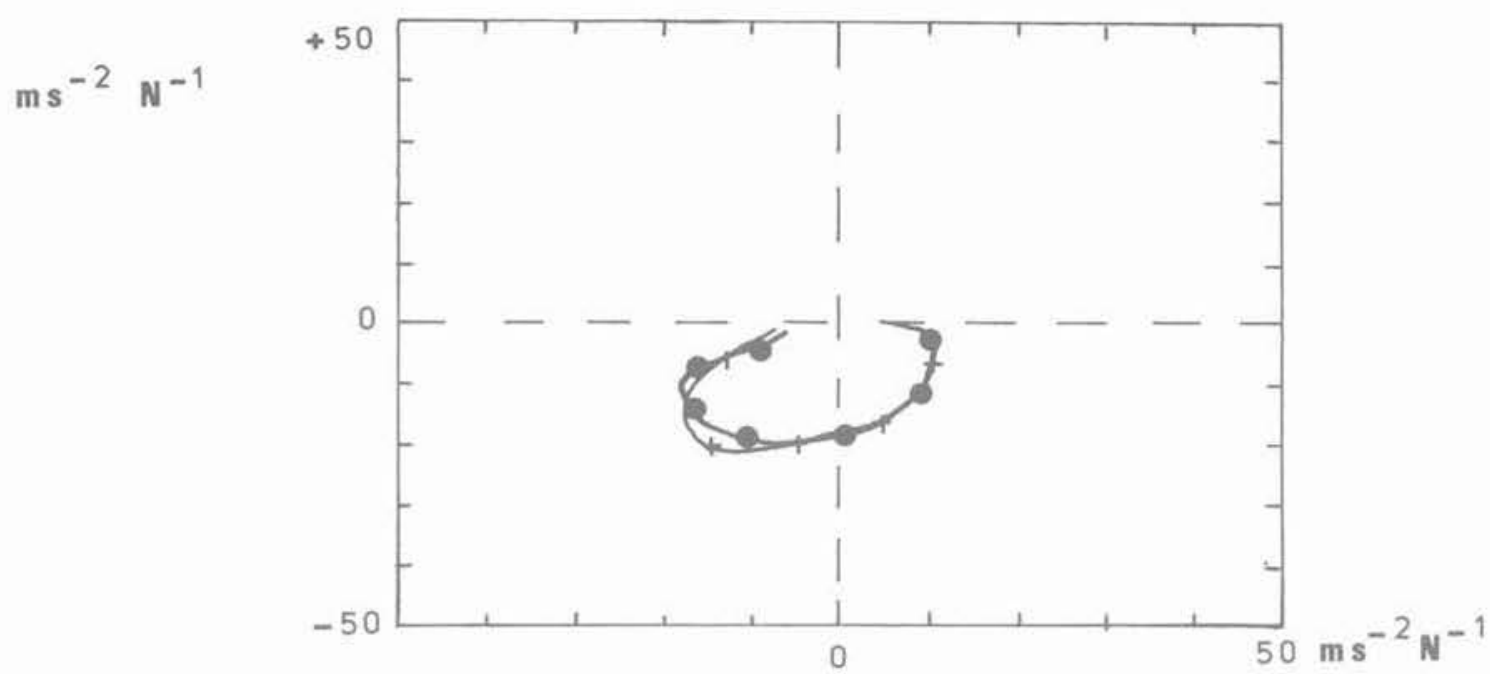

Fig. 17. - Lieu de Nyquist - Superposition de la FRF mesurée et de la FRF reconstituée en tenant compte de la partie non-causale.

Fig. 17. - Nyquist plot - Measured FRF and reconstructed FRF

superposition in which non causal part is taken into account.

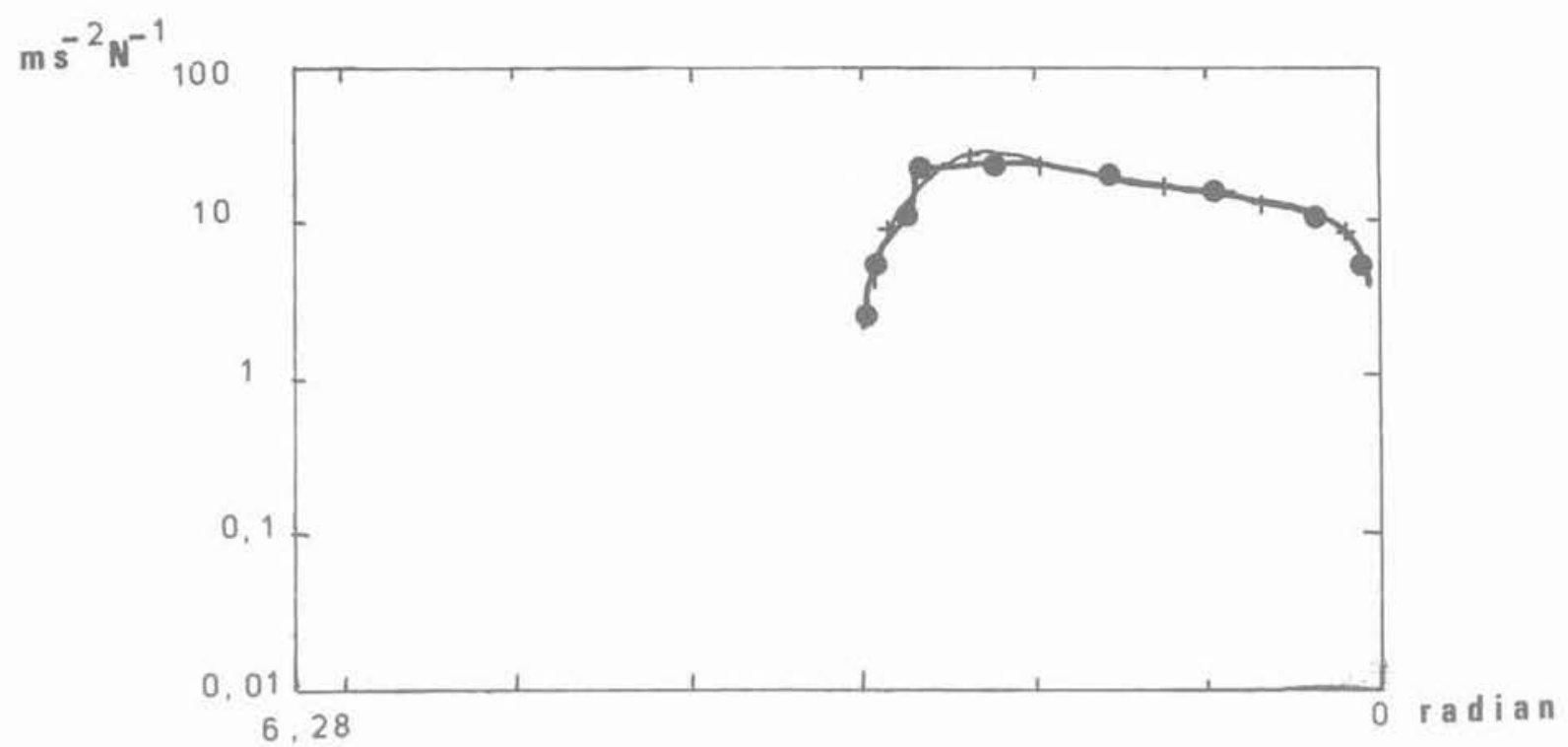

Fig. 18. - Diagramme de Nichols - Superposition de la FRF mesurée et de la FRF reconstituée en tenant compte de la partie non-causale.

Fig. 18. - Nichols plot - Measured FRF and reconstructed FRF superposition in which non-causal part is taken into account.

\subsection{Stratégies possibles en vue d'une modélisation}

\subsubsection{Reconstitution de la fonction expérimentale :}

VILLEDIEU [15] propose une reconstitution de la fonction mesurée à partir des paramètres noncausaux. Il peut paraître intéressant d'inverser la position naturelle des points de la figure 9 et de cons- truire une fonction sur la base de la partie non-causale à l'aide de la méthode MDOF. Cette fonction, reconstituée par les paramètres non-causaux extraits, est de nouveau inversée dans le domaine temporel. La superposition de la fonction reconstituée causale avec la fonction reconstituée non-causale, permet d'obtenir une fonction comparable à la fonction expérimentale. Dans le plan de Nyquist, ou dans le plan de Nichols, la reconstitution de la fonction mesurée est assez satisfaisante. 
Nous devons remarquer que cette reconstitution, si elle donne un résultat encourageant, n'est pas justifiée sur le plan théorique.

\subsubsection{Calcul des moments spectraux}

Nous avons utilisé le signe des moments spectraux afin de modéliser le type de non-linéarité rencontrée.

HAOUI [7] utilise l'expression des moments statistiques afin de classifier les types de non-linéarités. Suivant le signe des moments statistiques, il montre que l'on est en présence soit d'un frottement de Coulomb, d'un amortissement quadratique, d'une rigidité quadratique, etc. (tableau 1).

En utilisant la transformée de Hilbert comme référence, il caractérise l'écart entre la fonction mesurée et sa transformée de Hilbert. Cette différence est désignée par la fonction de distorsion $D(f)$ :

$D(f)=G(f)-H(f)$

Les moments statistiques peuvent être exprimés sous la forme générale:

$m_{D}^{n}=\int_{f_{1}}^{f_{2}} f_{n}|D(f)| d f$

$\mathrm{n}=0:$

$m_{D}^{0}=\int_{f_{1}}^{f_{2}}|D(f)| d f$

$\mathrm{m}_{\mathrm{D}}^{0}$ correspond à l'aire sous la courbe $\mathrm{D}(\mathrm{f})$

$\underline{n}=1$ :

$m_{D}^{1}=\frac{1}{m_{D}^{0}} \int_{f_{1}}^{f_{2}} f|D(f)| d f \quad f=\frac{m_{D}^{1}}{m_{D}^{0}}$

f est la fréquence du centre de masse.

$\underline{n}=2:$

$\sigma_{D}^{2}=\frac{1}{m_{D}^{0}} \int_{f_{1}}^{i_{2}}(f-\bar{f})^{2}|D(f)| d f$

$\sigma_{\mathrm{D}}$ est l'écart quadratique.

$n=3$;

$S_{D}=\frac{1}{\sigma_{D}^{3} m_{D}^{0}} \int_{f_{1}}^{f_{2}}(f-\bar{f})^{3}|D(f)| d f$

$\mathrm{S}_{\mathrm{D}}$ est le coefficient d'asymétrie. $\underline{n}=4$

$C_{D}=\frac{1}{\sigma_{D}^{4} m_{D}^{0}} \int_{f_{1}}^{f_{2}}(f-\bar{f})^{4}|D(f)| d f$

$C_{D}$ est le coefficient d'aplatissement ou de concentration.

Dans l'application de ces formules, il est important que la caractérisation s'effectue séparément sur la partie réelle et la partie imaginaire de la fonction $D(f)$, afin d'éviter la disparition des effets opposés. Si l'on veut obtenir des résultats comparables, il est indispensable que le critère de choix des bornes $f_{1}$ et $f_{2}$ soit identique pour tous les calculs. Nous avons choisi $f_{1}$ et $\mathrm{f}_{2}$ par rapport à la valeur de la fréquence de résonance $f_{T}$ pour le mode $f$.

$$
\begin{aligned}
& \mathrm{f}_{1}=0,8 \mathrm{f}_{\mathrm{r}} \\
& \mathrm{f}_{2}=1,2 \mathrm{f}_{\mathrm{s}}
\end{aligned}
$$

Ces bornes d'intégration, d'après BONNARD [2], exploitent au maximum l'information au niveau de la résonance.

Nous avons utilisé ces formules à laide du logiciel * HILTRA "; le tableau 2 donne les signes des moments spectraux pour les essais sur le spécimen en aluminium en sable saturé. Il est difficile, à partir des signes issus de nos fonctions mesurées, d'effectuer une corrélation avec le tableau 1 donné par HAOUI. Cela peut être expliqué par un couplage important des effets non-linéaires en raideur et en amortissement sur notre système sol-pieu. La disparité des signes est aussi valable pour les essais en sable sec.

\subsubsection{Identification des noyaux de Wiener}

Nous relatons les travaux de WIENER d'après l'ouvrage de SCHETZEN [14].

Cette méthode consiste à décomposer la fonctionnelle « entrée-sortie * du système étudié suivant les termes de séries, soit de Volterra, soit de Wiener.

Sur le plan mathématique, les conditions de convergence de la série de Volterra sont astreignantes; les fonctionnelles de la série de Volterra ne sont pas orthogonales entre elles; aussi, pour assurer la convergence, il faut que l'erreur tende vers zéro quand le nombre de termes tend vers l'infini, mais aussi que l'erreur concernant toutes les dérivées tende vers zéro.

WIENER ([14]) utilise les fonctionnelles non-homogènes.

On commence par définir la fonctionnelle de Volterra d'ordre zéro :

$\mathrm{H}_{0}[\mathrm{x}(\mathrm{t})]=\mathrm{h}_{0}$

$\mathrm{h}_{0}$ est une constante.

La fonctionnelle suivante est la fonctionnelle de Volterra non-homogène du premier degré, 
Tableau 1 - Résultats d'identification des non-linéarités par les moments statistiques (d'après HAOUI, ([I7]) Table 1 - Identification results of non-linearities by statistic moment (after Haoui).

\begin{tabular}{|c|c|c|c|c|c|c|c|c|}
\hline \multirow{2}{*}{$\begin{array}{c}\text { Moments } \\
\text { spectraux }\end{array}$} & \multicolumn{2}{|c|}{$\begin{array}{c}\text { Frottement } \\
\text { de Coulomb }\end{array}$} & \multicolumn{2}{c|}{$\begin{array}{c}\text { Amortissement } \\
\text { quadratique }\end{array}$} & \multicolumn{2}{c|}{$\begin{array}{c}\text { Rigidité } \\
\text { quadratique }\end{array}$} & \multicolumn{2}{c|}{$\begin{array}{c}\text { Hystérésis } \\
\text { non-linéaire }\end{array}$} \\
\cline { 2 - 8 } & $\mathrm{Re}$ & $\mathrm{Im}$ & $\mathrm{Re}$ & $\mathrm{Im}$ & $\mathrm{Re}$ & $\mathrm{Im}$ & $\mathrm{Re}$ & $\mathrm{Im}$ \\
\hline $\mathrm{m}$ & + & - & - & + & + & + & + & + \\
$\sigma_{2}$ & - & + & - & + & + & - & + & + \\
$\mathrm{s}$ & + & + & - & - & - & + & + \\
$\mathrm{c}$ & + & - & - & + & + & + & + \\
\hline
\end{tabular}

Tableau 2. Résultats pour les essais en sable saturé pieu aluminium Table 2 - Results of tests in satured sand-Aluminium pile.

\begin{tabular}{|c|c|c|c|c|c|c|c|c|c|c|c|c|}
\hline \multirow{3}{*}{$\begin{array}{l}\text { Moments } \\
\text { spectraux }\end{array}$} & \multicolumn{12}{|c|}{$\begin{array}{l}\mathrm{H} \text { : } \\
\text { Hauteur de la masse }\end{array}$} \\
\hline & \multicolumn{2}{|c|}{$H=33,5$} & \multicolumn{2}{|c|}{$H=30$} & \multicolumn{2}{|c|}{$H=26$} & \multicolumn{2}{|c|}{$H=22$} & \multicolumn{2}{|c|}{$H=18$} & \multicolumn{2}{|c|}{$H=14$} \\
\hline & $\operatorname{Re}$ & $\mathrm{Im}$ & $\mathrm{Re}$ & Im & $\operatorname{Re}$ & Im & $\operatorname{Re}$ & Im & $\mathrm{Re}$ & Im & $\mathrm{Re}$ & $\mathrm{Im}$ \\
\hline $\mathrm{m}$ & + & - & - & - & - & + & + & - & + & + & - & + \\
\hline$\sigma^{2}$ & + & - & + & - & + & + & + & + & + & + & + & - \\
\hline$s$ & + & t & + & - & + & - & + & - & - & - & t & + \\
\hline c & - & - & - & + & - & + & + & - & + & - & - & + \\
\hline
\end{tabular}

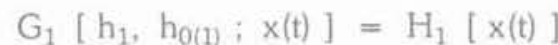

$+\mathrm{H}_{0(1)}[\mathrm{x}(\mathrm{t})]$

$G_{1}\left[h_{1}, h_{0(1)} ; x(t)\right]=\int_{-\infty}^{+\infty}$

$h_{1\left(\tau_{1}\right)} x\left(t-\tau_{1}\right) d \tau_{1}+h_{0(1)}$

$\mathrm{H}_{0(1)}$ est la fonctionnelle de Volterra d'ordre zéro.

En généralisant cette relation, la fonctionnelle nonhomogène d'ordre $\mathrm{p}$ s'écrit :

$g_{p}\left[h_{p}, h_{p-1(p)}, \ldots, h_{0(p)} ; x(t)\right]=\sum_{n=0}^{p} H_{n(p)}[x(t)]$ $g_{p}\left[h_{p}, h_{p-1(p)}, \ldots, h_{0(p)} ; x(t)\right]=$

$h_{0(p)}+\sum_{n=1}^{p} \int_{-\infty}^{+\infty} \ldots \int_{X\left(\varphi-\tau_{1}\right) \ldots}^{+\infty} h_{n(p)}\left(\tau_{1}, \ldots, \tau_{n}\right)$

$\mathrm{x}\left(\mathrm{t}-\tau_{\mathrm{n}}\right) \mathrm{d} \tau_{1} \ldots \mathrm{d} \tau_{\mathrm{n}}$

Les fonctionnelles $\mathrm{G}$ de Wiener sont définies par la somme des fonctionnelles non-homogènes de Volterra, telles que leur moyenne temporelle soit nulle pour un signal d'excitation aléatoire Gaussien :

$H_{m}[x(t)] g_{n}\left[k_{n}, k_{n-1(n)}, \ldots, k_{0(n)} ; x(t)\right]=0$

pour $\mathrm{m}<\mathrm{n}$ 
La fonction d'auto-corrélation de $\mathrm{x}(\mathrm{t})$ est donnée par :

$\phi_{x x}(\tau)=A \delta(\tau)$

$g_{n}\left[k_{n}, k_{n-1(n)}, \ldots, k_{0(n)} ; x(t)\right]$ est orthogonale à toute fonctionnelle de Volterra de degré strictement inférieur à $n$.

La réponse $y(t)$ d'un système non-linéaire soumis à une excitation $\mathrm{x}(\mathrm{t})$ peut s'écrire,

$y(t)=\sum_{p=0}^{\infty} G_{n}\left[k_{n} ; x(t)\right]$

$k_{n}$ est le noyau de Wiener d'ordre $n$.

SCHETZEN propose de générer des retards $\sigma_{1}$ par des méthodes numériques et d'effectuer un moyennage. La figure 19 montre la procédure permettant de déterminer le noyau de Wiener d'ordre $\mathrm{n}$ par des corrélations multiples avec un signal d'entrée $x(t)$ aléatoire :

$k_{n}\left(\sigma_{1}, \ldots, \sigma_{n}\right)=$

$\frac{1}{n ! A^{n}} \int_{-\infty}^{+\infty} \ldots \int_{-\infty}^{+\infty} h_{p}\left(\tau_{1}, \ldots \tau_{p}\right)$

$x\left(t-\tau_{1}\right) \ldots x\left(t-\tau_{p}\right) x\left(t-\sigma_{1}\right) \ldots x\left(t-\sigma_{n}\right) d \tau_{1} \ldots d \tau_{p}(3$

VINH a utilisé cette méthode à l'ordre deux pour des chocs programmés. Néanmoins, cette méthode reste limitée pour les noyaux d'ordre supérieur à deux par les temps de calcul nécessaires pour la détermination des corrélations multiples.
Ce procédé pourrait s'appliquer à la détermination des noyaux de Wiener d'un système « sol-structure » soumis à un séisme. En effet, on peut assimiler les accélérogrammes des séismes à des signaux d'entrée du type Gaussien et non-stationnaire (KREE [8]).

\subsubsection{Approximation bidimensionnelle par les polynômes de Tchebycheff}

L'étude des structures dynamiques linéaires repose sur le principe de superposition des équations différentielles à coefficients constants. L'équation étant résolue, la réponse de la structure peut être calculée explicitement en fonction du temps.

Pour les systèmes non-linéaires, les méthodes mathématiques, développées à partir des équations à coefficients non-constants, consistent, le plus souvent, à déterminer si le mouvement est périodique ou non, et, si oui, à calculer sa période. L'étude dans le plan de phase $(x, \dot{x})$ permet de donner les conditions d'équilibre du système (VOLTERRA [17]).

Il peut paraître intéressant d'obtenir expérimentalement un ensemble de points $\left(x_{1}, \dot{x}_{i}\right)$ et d'appliquer une méthode d'optimisation en vue d'identifier les termes de couplage non-linéaire. La méthode d'identification présentée repose sur la recherche des formes approchées des forces internes du système. ARGOUL [1] propose une technique utilisant l'approximation bidimensionnelle par les polynômes de Tchebycheff à l'aide d'un procédé itératif.

Pour un système dunamique discrétisé à $\mathrm{n}$ degrés de liberté, on définit les forces internes $f$ à partir de l'équation du mouvement :

$f(x, \dot{x})=p(t)-m \ddot{x}(t)$

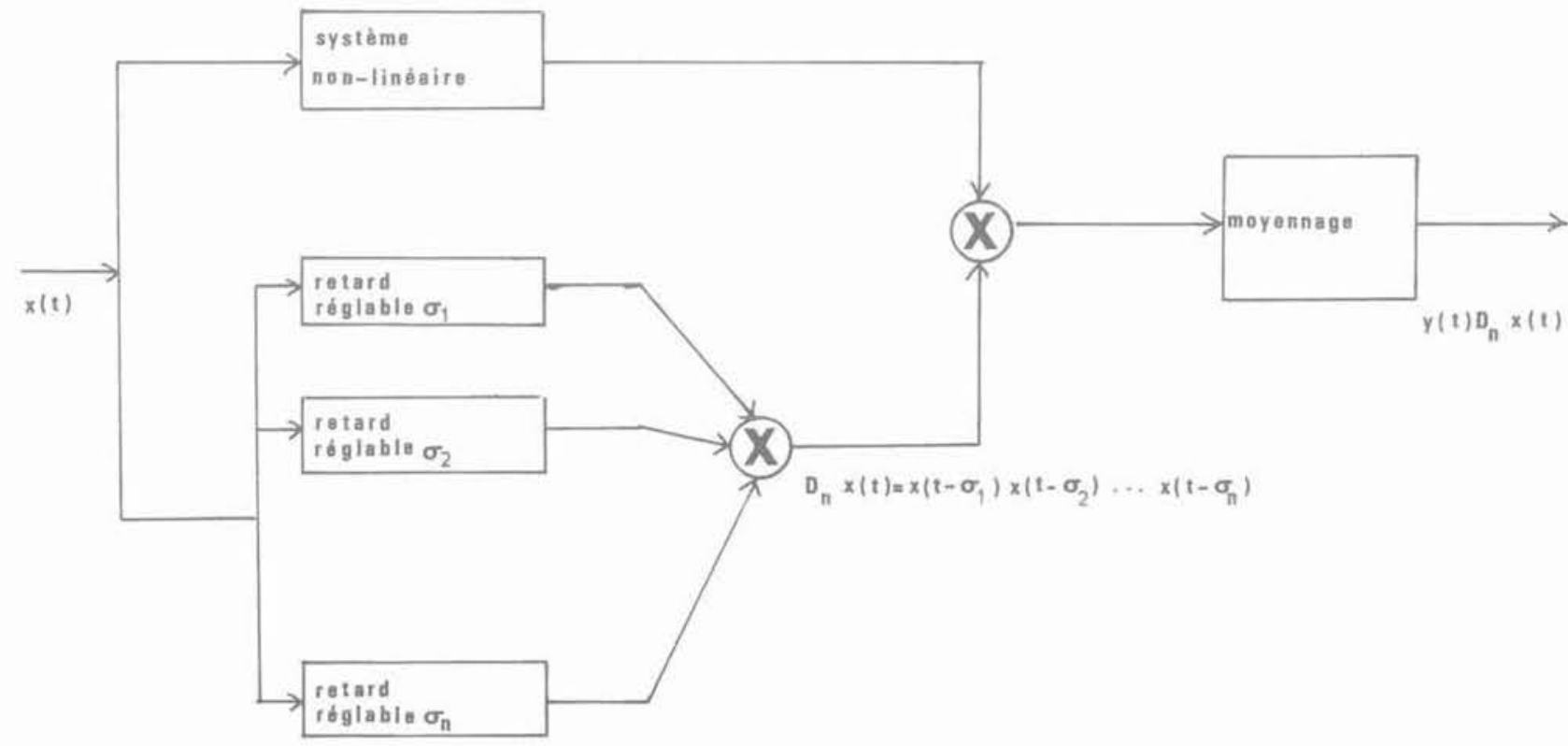

Fig. 19. - Détermination des noyaux de Wiener, d'après Schetzen (14). Fig. 19. - Wiener kernel determination, after Schetzen (14). 


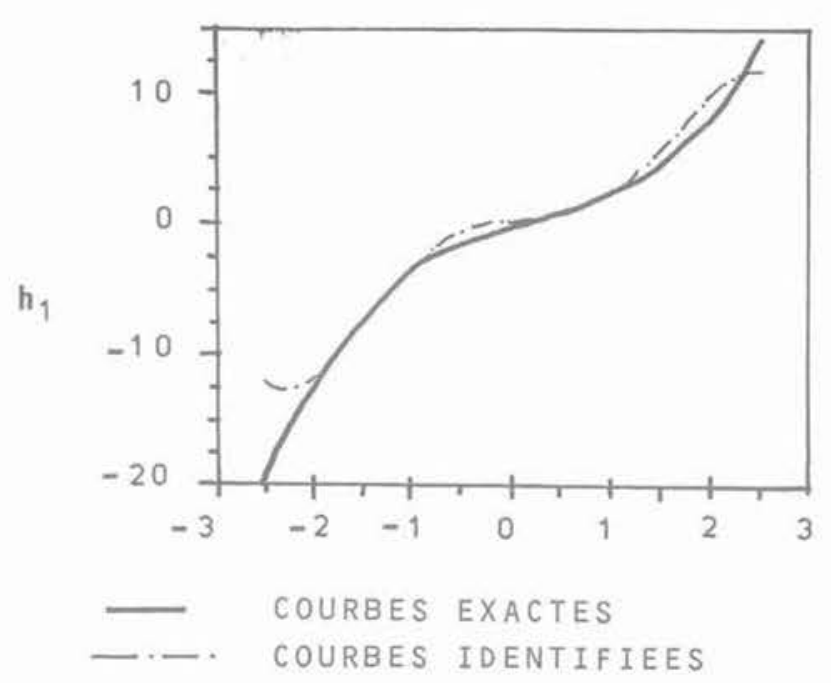

Fig. 20. - Cas d'une rigidité cubique, d'après Argoul [1] Courbes exactes

Courbes identifiées.

$m$ représente la matrice de masse d'ordre $n$, $\ddot{x}(\mathrm{t})$ est le vecteur accélération accessible à partir de mesures sur une période $T_{\max }$,

$p(t)$ est le vecteur force d'excitation.

En accord avec les méthodes de l'analyse modale, on utilise une représentation du type :

$h(\mathrm{u}, \dot{\mathrm{u}})=\mathrm{P}(\mathrm{t})-\ddot{\mathrm{u}}(\mathrm{t})$

où

et

$$
\begin{aligned}
& \mathrm{h}=\Phi^{\mathrm{T}} \mathrm{f} \\
& \mathrm{P}=\Phi^{\mathrm{T}} \mathrm{p} \\
& \ddot{\mathrm{u}}=\Phi^{\top} \mathrm{m} \ddot{\mathrm{x}}
\end{aligned}
$$

$\Phi^{T}$ est le vecteur transposé de $\Phi ; \Phi$ représente une estimation des $r$ modes normaux linéarisés prédominants et identifiés à l'aide d'essais vibratoires effectués à un faible niveau d'amplitude.

Pour une excitation donnée $\mathrm{p}(\mathrm{t})$, on connaît expérimentalement les $r$ coordonnées $h$ de $h$ en certains points de l'espace d'état $\epsilon$. On fait l'hypothèse liée à la représentation modale que $h_{\mathrm{j}}(\mathrm{u}, \mathrm{u})$ se décompose en la somme d'un terme prépondérant $h_{1}^{1}\left(u_{1}, \dot{u}\right)$ représentant la contribution à $h$ du mode $i$, et de termes $h^{S}(S>1)$ dus à l'interaction des modes $j(j$ $\neq$ i) avec le mode $\mathrm{i}$.

A partir de la connaissance expérimentale de $h_{j}(u, u)$. on approche $h_{1}^{1}$ exprimé en termes d'une double série de polynômes de Tchebycheff. On suppose que l'erreur résiduelle $\left[h_{1}(\mathrm{u}, \dot{u})-h^{1}(\mathrm{u}, \dot{\mathrm{u}}]\right.$ due à l'approximation précédente constitue une approximation de $h_{i}^{2}\left(u_{i}, u_{i}\right)$ que l'on développe en polynômes de Tchebycheff. On opère de cette manière jusqu'au critère d'arrêt pour $\epsilon$ petit fixé :

$$
\left\|h_{i}(u, \dot{u})-\sum_{s} h_{i}^{s}\right\|<\epsilon
$$

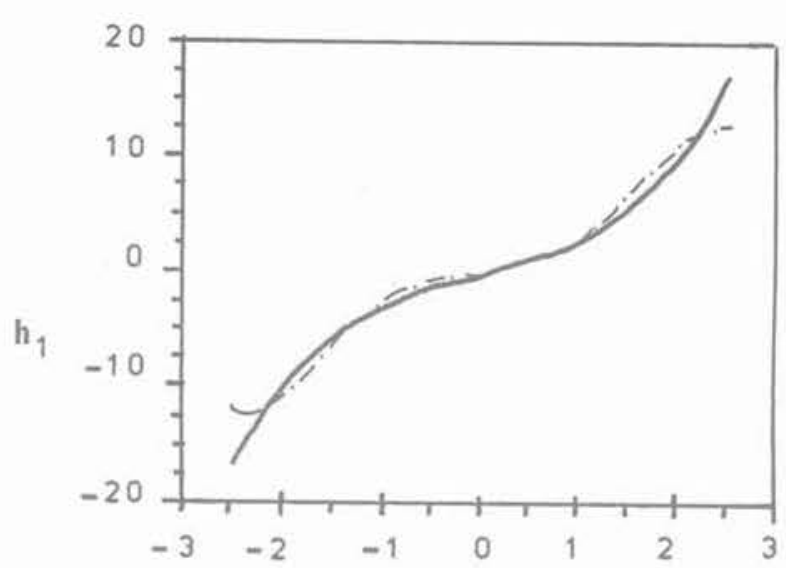

Fig. 20. - Case of cubic stiffness, after Argoul [1]
Exact curves
Identified curves.

On obtient les coefficients des polynômes par une intégrale double calculée numériquement. Une difficulté vient du fait que même si l'on a choisi un type d'excitation convenable et une période $T_{\max }$ suffisante, il existe des zones de l'espace de phase où la densité des points de mesure est trop faible pour permettre une bonne interpolation.

ARGOUL [1] propose d'utiliser une affinité qui permet une extrapolation du domaine où les points de mesure sont nombreux vers le domaine où l'information est clairsemée. ARGOUL a testé le procédé sur un système à deux degrés de liberté avec un élément non-linéaire en $\alpha u^{3}$ entre les deux masses. Ces résultats sont présentés sur la figure 20 .

\section{CONCLUSION}

Après le développement spectaculaire des méthodes d'identification linéaire, il subsiste des difficultés pratiques compromettant la validité de lidentification modale lorsque des non-linéarités sont rencontrées.

L'identification des caractéristiques dunamiques peut être améliorée en mettant en œuvre les méthodes existantes. En pratique, il en résulte un surcroît de complexité de l'essai de vibration, puisqu'il faut déceler les défauts de linéarité par des tests vibratoires préalables, choisir la stratégie la mieux adaptée et l'appliquer.

Dans quelques années, les systèmes d'analyses dynamiques devront comporter les moyens et les codes de calcul nécessaires pour la mise en œuvre des stratégies adaptées aux types de non-linéarités rencontrés en pratique.

Dans cette présentation consacrée à l'étude des nonlinéarités, l'utilisation de la transformée de Hilbert a permis de détecter les non-linéarités rencontrées lors 
de nos essais de vibrations. Nous avons tenté, par des analogies, de mettre en évidence l'influence de la plasticité, de la variation de la raideur et de la variation de l'amortissement sur les fonctions de réponse en fréquence grâce à une analyse détaillée des diverses représentations possibles d'une fonction à variable complexe.

Des stratégies pour identifier les paramètres nonlinéaires actuellement développées sont présentées. Des logiciels permettant le calcul des corrélations multiples autorisent l'évaluation des fonctions de transfert multidimensionnelles dans le cadre des théories de Volterra et de Wiener.

Les techniques d'approximation bidimensionnelle dans le plan de phase permettent une identification des termes de couplage non-linéaires.

Les essais combinés « centrifugation-vibration « associés à des techniques modernes de traitements des signaux autorisent une simulation scientifiquement acceptable des efforts sur les systèmes * sol-structure ", et une caractérisation des paramètres physiques permettant la validation des lois de comportement en dynamique des sols.

\section{BIBLIOGRAPHIE}

[1] ARGOUL P. (1987). Une méthode d'identification des structures non-linéaires en dynamique, $8^{e}$ Congrès français de mécanique, Nantes. pp. 66-67.

[2] BONNARD R. (1987), «Comportement nonlinéaire des structures - Simulation numérique et caractérisation par les moments spectraux des non-linéarités », Thèse C.N.A.M., Conservatoire National des Arts et Métiers, Paris.

[3] BOURDIN B. (1987), "Vibrations horizontales de pieux en centrifugeuse ", Thèse de l'Université de Bordeaux $1, \mathrm{n}^{\circ} 174$.

[4] BOURDIN B.. MORLIER P. (1988), "Caractérisation des non-linéarités dans un essai de vibra. tion sur fondation ». Centrifugeuse' 88 - Congrès International sur la Modélisation Géotechnique en Centrifugeuse, 25-27 Avril 1988, Paris.

[5] CHAN X.B., KOBUS J.M. (1987), « Etude des efforts du second ordre sur une structure soumise à une houle aléatoire: Application du cas d'un cylindre fixe $», 8^{e}$ Congrès français de mécanique, pp. 410-411, Nantes.
[6] FRECHET M. (1910), «Sur les fonctionnelles continues ", Annales Scientifiques de l'Ecole Normale Supérieure, (3), vol. 27, pp. 193-216, Paris.

[7] HAOUI A. (1984), «Transformée de Hilbert et applications aux systèmes non-linéaires ». Thèse de Docteur-Ingénieur - Institut Supérieur des Matériaux et de la Construction Mécanique, Paris.

[8] KREE P. SOIZE C. (1983), « Mécanique Aléatoire ". Editions Dunod, Chapitre 7, pp. 287-355.

[9] LALLEMENT G., FILLOD R., PIRANDA J. (1982) "Identification des structures nonlinéaires. Application au calcul des comportements dynamiques par synthèse modèle ", Rapport final d'une recherche financée par la Direction Générale de la Recheche et de la Technologie, $n^{\circ}$ 82-S-1083

[10] LUONG M.P. (1986), "Simulation des forces de masse ", Annales I.T.B.T.P., n 42, Série «Essais et Mesures », 204, pp. 111-222 (Février 1986).

[11] LUONG M.P., BONAZ R., PECKER A. et BOURDIN B. (1988), « Pieux et groupes de pieux soumis à des sollicitations forcées horizon. tales en centrifugeuse ", Centrifugeuse' 88 , Congrès international sur la modélisation géotechnique en centrifugeuse, 25-27 Avril 1988, Paris, Balkema, pp. 505-513.

[12] NORMAN F., HUNTER Jr (1985), "An inves. tigation of the time history and modal responses of some simple linear and non linear systems ", Proc. of the 3rd Int. Modal Analysis Conf. Orlando, pp. 410-418.

[13] OKUBO N. (1982), * The effect of non-linearity on transfer function measurement ", Sound and Vibration, pp. 34-37 (Novembre).

[14] SCHETZEN M. (1980), * The Volterra and Wiener theories of non linear systems». Wiley Interscience Publication.

[15] VILLEDIEU C. (1985), *Un moyen rationnel pour supprimer la partie non-causale d'une fonction réponse ». Proceedings of the Tenth Int. Seminar on Modal Analysis, Part IV, Section A3.

[16] VINH T., HAOUI A., FEI B.J., CHEVALIER Y. (1984), "Extension de l'analyse modale aux sustèmes non-linéaires par la transformée de Hil. bert », Journal de Matériaux, Mécanique, Electricité, n० 404-405-406, pp. 5-14.

[17] VOLTERRA E., ZACHMANOGLOU (1965), "Dynamics of Vibrations », Charles E. Merril Books, Inc. Columbus, Ohio - Chapitre 5, pp. 439-512. 